\title{
Genome-wide analysis and expression profiles of the StR2R3-MYB transcription factor superfamily in Solanum Tuberosum
}

\section{Yuanming Li}

Gansu Agricultural University

Kui Lin-Wang

Plant and Food Research

Zhen Liu

Gansu Agricultural University

Andrew C. Allan

Plant and Food Research

Junlian Zhang

Gansu Agricultural University

Yuhui Liu ( $D$ lyhui@gsau.edu.cn )

Gansu Agricultural University https://orcid.org/0000-0002-3095-5858

\section{Research article}

Keywords: potato, MYB transcription factor, Expression profiles, Anthocyanin biosynthesis, Drought stress, EAR

Posted Date: May 18th, 2019

DOl: https://doi.org/10.21203/rs.2.9669/v1

License: (9) This work is licensed under a Creative Commons Attribution 4.0 International License. Read Full License 


\section{Abstract}

Background: MYB transcription factors comprise one of the largest families in plant kingdom, which play a variety of functions in plant developmental processes and defence responses. However, it has not been systematically studied in Potato (Solanum tuberosum), which is the most important non-cereal crop world-wide. Results: In the present study, a total of 108 StR2R3-MYB transcription factors were identified and further phylogenetically classified into 28 subfamilies, as supported by highly conserved gene structures and motifs. Collinearity analysis showed that the segmental duplication events played a crucial role in the expansion of StR2R3-MYB gene family. Synteny analysis indicated that 37 and 13 StR2R3-MYB genes were orthologous to Arabidopsis and wheat, respectively, and these gene pairs have evolved under strong purifying selection. RNA-seq data from different tissues and abiotic stresses revealed tissue-preferential and abiotic stress-responsive StR2R3-MYB genes. We further analyzed StR2R3-MYB genes might be involved in anthocyanin biosynthesis and drought stress by using RNA-seq data of pigmented tetraploid potato cultivars and drought-sensitive and -tolerant tetraploid potato cultivars under drought stress, respectively. Moreover, EAR motifs were found in 21 StR2R3-MYB proteins and 446 pairs of proteins were predicted to interact with 21 EAR motif-containing StR2R3-MYB proteins by constructing the interaction network with medium confidence (0.4). Additionally, Gene Ontology (GO) analysis of the 21 EAR motif-containing StR2R3-MYB proteins was performed to further investigate their functions. Conclusions: In this work, we systematically identified StR2R3-MYB genes by analyzing the potato genome sequence using a set of bioinformatics approaches. Genome-wide comparative analysis of StR2R3-MYB genes and their expression analysis identified members of this superfamily may be involved in tissue-specific development, anthocyanin biosynthesis and abiotic stress responses.

\section{Background}

Transcription factors are important regulators of gene expression by involving chromatin organization, DNA methylation, dimerization and sequence-specific DNA binding to control many aspects of plant for responding to abiotic and biotic stresses and modulate developmental and metabolic processes through activating or suppressing of target genes [1,2]. Transcription factors can be classified into many different families on the basis of DNA-binding domain in the regulatory regions of downstream target genes [3].

The MYB transcription factors (TFs) comprise one of the largest TF families in the plant kingdom, are distinguished by the highly conserved MYB domain [4, 5]. MYB family members are divided into four subfamilies depending on the number of repeat ( $s$ ) in the MYB domain, which consists of 1-4 imperfect tandem repeats in N-terminus, including 1R-MYB, R2R3-MYB, 3R(R1R2R3)-MYB and 4R-MYB with one, two, three and four MYB repeats [5, 6], of which, 1R-MYBs were referred to as MYB-related proteins [7]. Generally, the R2R3-MYB members are the predominant form found in higher plants [2]. The MYB repeat is approximately 50-53 amino acid residues in length, containing three regularly spaced tryptophan (or aliphatic) residues that together form a helix-turn-helix $(\mathrm{HTH})$ fold $[8,9]$. The second and third a-helix of each repeat interacts with the major DNA groove at the specific recognition site C/TAACG/TG during 
transcription $[10,11]$. On the contrary, the $\mathrm{C}$-terminal modulator region is highly divergent that is responsible for the regulatory activity of the MYB proteins [12].

The MYB TF family is present in all eukaryotes. The first gene described as encoding a MYB domaincontaining protein was "Oncogene" $v$-MYB identified in avian myeloblastosis virus (AMV) [13], subsequently, human c-MYB proto-oncoprotein and two vertebrate MYB TFs A-MYB and B-MYB were found and proved to take part in the regulation of cell differentiation, proliferation, and apoptosis [14, 15]. In plants, Zea mays $\mathrm{C} 1$, a homolog of mammalian $c-M Y B$ gene, is responsible for the regulation of anthocyanin biosynthesis [16]. C1 was the first plant TF as well as the first plant $M Y B$ gene to be cloned and functionally characterized.

Since the first plant MYB gene was characterized, an increasing number of plant MYB TF family members have been identified and characterized in a wide range of plant species such as in Arabidopsis thaliana [5], Brassica napus [17], Solanum lycopersicum [18], Populus trichocarpa [19], Maize [20], Soybean [21], Apple [22] and Pineapple [23]. Furthermore, multiple MYB proteins in plants have been functionally investigated in detail. The results from these works have proved that MYB proteins, especially the R2R3-MYB proteins, play an important role in many biological processes including primary and secondary metabolism, cell fate and identity, cell development and cell cycle, response to various biotic and abiotic stresses, and hormone synthesis and signal transduction [5, 24-26]. For example, a number of MYB TFs regulating anthocyanin biosynthetic pathway have been identified from many species, such as AtMYB75 (or PAP1), AtMYB90 (or PAP2), AtMYB113 and AtMYB114in Arabidopsis [27, 28], MdMYBA,MdMYB1, MdMYB10 and MdMYB110ain apple [29, 30], SIMYB12 in tomato [31] and StAN1 in potato [32,33]. An amount of MYB TFs involved in regulating responses to environmental stresses were also studied such as GbMYB5 confers drought tolerance in cotton and transgenic tobacco plants [34], LcMYB1 confers salt tolerance in transgenic Arabidopsis [35], overexpression of StMYB1R-1 in potato plants improved plant tolerance to drought stress [36].

Potato (Solanum tuberosum L.), originated from the Andean regions of Peru and Bolivia [37], is now grown worldwide as the third most important food crop plant after wheat and rice. In recent decades, pigmented potato cultivars attracted a lot of attention from consumers and researchers worldwide, as it is a rich source of anthocyanins, in particular acylated derivatives [38], which not only have key roles in protection against UV radiation and cold temperatures, and response to drought stress [39-41], but also have potential health benefits such as protection against some cancers and neuronal and cardiovascular diseases in humans [42].

Potato is very sensitive to drought with yield reduction beginning under moderate deficit of soil moisture $[43,44]$, genetic and molecular biological approaches were used to overcome potato yield loss under drought stress [36, 45-48], however, there is still little known about drought stress tolerance mechanisms in potato plants.

Since R2R3-MYB TFs play an important role in wider plant biological processes, and were studied in a numerous plant species, but there are only very limited reports on the functional characterization of R2R3- 
MYB TFs in potato $[32,33,36,46,49,50]$. Thus it is meaningful to uncover the R2R3-MYB TFs' function, evolution and expression profiles in potato based on the published sequence data [51].

In the current study, we totally identified an expanded StR2R3-MYB family with 108 members, and comprehensive analysis of the phylogenetic relationships, sequence features, gene duplications, chromosome distribution, motif recognition were further investigated. In addition, we performed a comprehensive expression analysis of StR2R3-MYB genes in different tissues and under abiotic stresses in doubled monoploid (DM) potato, as well as in white and pigmented potato cultivars and in droughtsensitive and -tolerant cultivars of tetraploid potato using RNA-seq data to identify StR2R3-MYB genes closely associated with spatial distribution, anthocyanin biosynthesis and multiple stresses. Moreover, 21 StR2R3-MYB genes containing EAR motif were obtained and an interaction network between these EAR motif-containing R2R3-MYBs with other potato genes were constructed. Additionally, Gene Ontology (GO) analysis of the 21 EAR motif-containing StR2R3-MYB proteins was performed to investigate their functions. The findings should inform the characterization of StR2R3-MYB superfamily and provide valuable information for further functional elucidation of these genes in potato.

\section{Methods}

Identification of potato MYB family genes

Hidden Markov Model (HMM) profile for the MYB binding domain (PF00249) was downloaded from the Pfam database (http://pfam.xfam.org/) [52] and used it as a query to search potato genome from the Potato Genome Sequencing Consortium (PGSC, http://solanaceae.plantbiology.msu.edu/pgsc_download.shtml) by HMMER 3.1

(http://hmmer.org/download.html) with a cutoff value of 0.01 . Additionally, 126 R2R3-MYB superfamily protein sequences in Arabidopsis thaliana were obtained from The Arabidopsis Information Resource (TAIR; http://www. arabidopsis.org/) based on previous work [2, 53]. The Pfam database, SMART website (http://smart.embl-heidelberg.de) [54] and the NCBI-CDD

(http://www.ncbi.nlm.nih.gov/Structure/cdd/wrpsb.cgi) [55] were used to confirm the obtained MYB proteins. All potato R2R3-MYB proteins were manually inspected to ensure that the gene models contained two MYB domains.

Sequence analysis and structural characterization of StR2R3-MYB genes

The exon-intron organizations of the StR2R3-MYB genes, including intron distribution patterns, phases and intro-exon boundaries, were graphically displayed by the Gene Structure Display Server (GSDS2.0 (http://gsds.cbi.pku.edu.cn/) [56] using the CDS and genome sequence of StR2R3-MYB genes. The conserved motifs of StR2R3-MYB TFs was predicted by using the MEME Suite web server (http://memesuite.org/tools/meme) analysis [57], with the following parameters: the maximum number of motifs was set to identify 20 motifs and optimum width of motifs was set from six to 100 amino acids.

Analyses of chromosome distribution, gene duplication and synteny for StR2R3-MYB genes 
The chromosome distribution information of the StR2R3-MYB genes were obtained from the database of potato genome downloaded from PGSC. MapChart software [58] was used for graphical presentation of StR2R3-MYB gene's chromosomal location. Tandem duplication and segmental duplication between potatogenes as well as the synteny block of the orthologous $R 2 R 3-M Y B$ genes between potato and Arabidopsis, wheat was obtained by using Multiple Collinearity Scan toolkit (MCscanX) (http://chibba.pgml.uga.edu/mcscan2/) [59] and visualized using the circos v0.69 [60]. To further estimate duplication events of StMYB genes, the synonymous (Ks) and nonsynonymous (Ka) were calculated by using KaKs_Calculator 2.0 (https://sourceforge.net/projects/kakscalculator2/) [61].

Phylogenetic analysis and classification of potato StR2R3-MYB proteins

The full-length amino acid sequences of StR2R3-MYB and AtR2R3-MYB proteins derived from PGSC and Ensembl Plants database (http://plants.ensembl.org/index. html) were used for phylogenetic analysis. Multiple sequence alignments of these R2R3-MYB proteins were performed using Clustal $\mathrm{X}$ with default parameters. An unrooted neighbor-joining $(\mathrm{NJ})$ phylogenetic tree was constructed using MEGA 7.0 software [62], with the following parameters: Poisson model; pairwise deletion; and 1000 bootstrap replications. The potato R2R3-MYB proteins were classified into different groups according to the topology of phylogenetic tree.

Plant materials and treatments

A purple potato cultivar 'Heimeiren' (HM, purple skin and purple flesh), a white potato cultivar 'Xindaping' (XD, white skin and white flesh) and a red potato cultivar 'Lingtianhongmei' (LT, red skin and red flesh) were grown in a greenhouse at Gansu Agricultural University, China. HM and XD are local cultivars in Gansu province, LT was cultivated by Potato Research Center of Inner Mongolia Academy of Agricultural and Animal Husbandry Sciences and Inner Mongolia Lingtian Biotechnology Co., Ltd., China. Six fresh tubers (diameter 4-5cm) were harvested, and cleaned with sterilized water. Skin tissue was carefully separated from cortex tissue using a scalpel to minimize flesh contamination, flesh tissue was isolated with at least $5 \mathrm{~mm}$ distance from the skin to eliminate skin contamination. The skin and flesh of these potatoes were then immediately frozen in liquid nitrogen and stored in a $-80^{\circ} \mathrm{C}$ freezer for later use.

For drought stress, a drought-sensitive cultivar 'Atlantic' (A) and a drought-tolerant cultivar 'Qingshu No.9' (Q) were field-grown with rainproof shed at Dingxi Academy of Agricultural Science, Gansu. A was cultivated by Dingxi Potato Research Institute and Q was cultivated by Qinghai Academy of Agriculture and Forestry Sciences of Qinghai Province, China. Two treatments were performed: drought stress and watered control with a drip irrigation system to control of watering. Each treatment had a randomized complete block design with three blocks (replications) and ten plants per block. For the first 4 weeks, all plants in both treatments were watered optimally and equally, after seedling, the plants under drought stress treatment went unwatered for whole growth period, while the plants under control treatment were still irrigated optimally during whole growth period until the foliage began to die naturally. The foliage of three pooled plants for each replicate was collected at early flowering stage, full-blooming stage and 
flower-falling stage, respectively, and then immediately frozen in liquid nitrogen and stored in a $-80^{\circ} \mathrm{C}$ freezer for later use.

RNA isolation and quantitative real-time RT-PCR

Total RNA extractions of skin and flesh from three potato cultivars, and of leaves from two potato cultivars, were carried out using the PureLink Plant RNA Reagent Kit (Invitrogen, USA) according to the manufacturer's instructions. The RNA was monitored by gel electrophoresis and quantified by using a Nanodrop ND-1000 spectrophotometer (Nanodrop Technologies, USA). Elimination of genomic DNA contamination and first-strand CDNA synthesis were carried out using oligo dT according to the manufacturer's instructions (SuperScript III, Invitrogen, USA). qRT-PCR was conducted on CFX96 Touch ${ }^{\text {TM }}$ Real-Time PCR Detection System (Bio-Rad, CA, USA) using SYBR ${ }^{\circledR}$ Premix Ex Taq ${ }^{\text {TM }}$ II (Takara Bio, Inc., Japan). qPCR conditions were as follows: $30 \mathrm{~s}$ at $95^{\circ} \mathrm{C}$, followed by 40 cycles of $5 \mathrm{~s}$ at $95^{\circ} \mathrm{C}, 30 \mathrm{~s}$ at 60 ${ }^{\circ} \mathrm{C}$, followed by $65-95^{\circ} \mathrm{C}$ melting curve detection. The qPCR efficiency of each gene was obtained by analysing the standard curve of a cDNA serial dilution. StEF-1a (AB061263) [63] was used for template normalization. Relative abundance was calculated with comparative $\mathrm{Ct}\left(2^{-\Delta \Delta} \mathrm{Ct}\right)$ method. The primers are listed in Additional files 1: Table S1.

RNA-Seq data analysis

Total RNA of the aforementioned samples with three biological replicates was chosen for further RNA-seq library construction. Next-Generation Illumina sequencing were performed by Biomarker Technologies Corporation (Beijing, China). High quality clean reads were obtained by trimming the raw reads filtering out contaminants, adapters, phred scores less than 20 and uncertain bases $\mathrm{N}$. The cleaned data were aligned to PGSC_DM_v3.4 gene models downloaded from PGSC by Bowtie2 (v2.2.9). The number of mapped clean reads were counted (by fragment) and subjected to differential expression analysis using the edgeR package (http://www.r-project.org/). Genes with the absolute value of log2FC (fold change) not less than 1 and a false discovery rate (FDR) $<0.05$ was considered as significant DEGs.

DEGs were annotated against nonredundant database (nr), SwissProt/UniProt Plant Proteins, KEGG, SwissProt/UniProt Plant Proteins, COG/KOG (Cluster of Orthologous Groups of proteins) and potato protein database (ftp:// ftp.jgi-psf.org/pub/compgen/phytozome/v9.0/Stuberosum) by BLASTX and the cut-off E-value was 1.0e-5. DEGs were then subjected to enrichment analysis of GO functions and KEGG pathways.

The Illumina RNA-seq data were also downloaded from the PGSC to study the expression patterns of MYB genes in various tissues and stress treatments. TBtools software [64] was used to generate the heatmap.

Identification of EAR motif-containing StR2R3-MYB proteins 
The StR2R3-MYB proteins were manually searched to identify candidate genes containing motifs DLNxxP or LxLxL [65]. Specific protein interactions were constructed by using online STRING v11.0 software (Search Tool for the Retrieval of Interacting Genes/Proteins, http://string-db.org/) [66] with combined score $\mathbb{4 0 0}$ (medium confidence). The interaction network was visualized by Cytoscape v3.7.1 [67]. The GO enrichment analysis was conducted by using the topGO package in R project.

\section{Availability of supporting data}

The raw data of the transcriptome analysis used in this study was submitted to the Sequence Read Archive (SRA) at NCBI under Project ID PRJNA528685 and PRJNA529980, and the expression data was also available Potato Genome Sequencing Consortium (PGSC, http://solanaceae.plantbiology.msu.edu/pgsc_download.shtml). The accession number and the website listed above were publicly available. The databases used in this study were publicly accessible and no special permissions were required.

\section{Results}

Identification and classification of the potato $M Y B$ genes

To identify the putative MYB proteins in potato genome, a hidden Markov model (HMM) search was performed using the HMM profile of the MYB binding domain against the potato genome downloaded from PGSC database. This HMM search resulted in identification of 319 potato gene models (DMGs). Subsequently, the Pfam, SMART website and NCBI-CDD analyses were performed to verify the presence of the MYB domains. Finally, a total of 233 non-redundant potato proteins were identified, including 108 R2R3-MYB proteins (2R-MYB) (Additional files 2: Table S2), 121 MYB-related proteins (1R-MYB) proteins, 3 R1R2R3-MYB proteins (3R-MYB) and $14 R-M Y B$ proteins. The lengths of StR2R3-MYB ranged from 136 to 523 amino acids, the molecular weights were between $1.61 \mathrm{kDa}$ and $5.73 \mathrm{kDa}$ and the predicted pl values ranged from 4.59 to 9.99 .

The functions of several Arabidopsis MYB proteins, especially the R2R3 MYBs, have been well characterized, and several functional clades have been identified by phylogenetic analysis of Arabidopsis MYB proteins [5]. To further understand the evolutionary relationship of R2R3-MYB proteins in potato and Arabidopsis and speculate the putative functions of the potato R2R3-MYB proteins based on the functional clades which have been identified in Arabidopsis, unrooted Neighbor-Joining (NJ) phylogenetic trees were constructed based on R2R3-MYB subfamilies (Fig. 1). The resulting trees generated 31 clades for R2R3-MYB subfamilies (named C1-C31) according to the sequence similarity and topology. Remarkably, 27 clades included different numbers of R2R3-MYB proteins from the two species, while two clades (C7 and C15) only contained Arabidopsis R2R3-MYB members, two clades (C23 and C24) only contained potato R2R3-MYB members. Additionally, an unrooted phylogenetic tree of StR2R3-MYB TFs was also constructed individually, as shown in Fig. 2A, 28 clades for R2R3-MYB 
subfamilies (named A1-A28) were generated, and 67 R2R3-MYB TFs were classified into 16 subgroups based on the phylogenetic analysis of Arabidopsis and potato in Figure 1.

Structure of StR2R3-MYB genes and conserved motifs

Since the analyses of gene structural diversity might be helpful for understanding the gene functions and evolution, the structural diversity of StR2R3-MYB genes was also investigated (Fig. 2B). Three members in A1 contained more exons than other subfamilies with 9-11 exons, four StR2R3-MYB genes (PG0007994, PG0024983, PG0003316 and PG0034577, which belonged to SG22) only have one exon, furthermore, the results showed that exon/intron structures were highly conserved in the same subfamilies of StR2R3-MYB genes, suggesting these conserved features play crucial roles for groupspecific functions (Fig. 2B).

We used online program MEME to search for conserved motifs shared by these StR2R3-MYB proteins to further study the diversification of these StR2R3-MYB genes in potato. In total, 20 conserved motifs were identified and designate as motif 1 to 20 (Fig. 2C). The details of the 20 motifs were referred in Additional files 3: table S3. Most of R2R3-MYB protein sequences had four highly conserved motifs and conserved motif orders. 107 out of 108 R2R3-MYB protein sequences all had motif 2, motif 15 , motif 1 , and motif 3 in that order, containing 23,11,50, and 18 amino acids, respectively, motifs 2,15 and left part of motif 1 were composed of the R2 repeat, and right part of motif 1 and motif 3 were composed of the R3 repeat. In addition to the highly conserved motifs composed of MYB domains (R2 and R3 repeats), other conserved motifs were also found in most of the closely related members (Fig 2C and Additional files 3: Table S3), suggesting possible functional similarity among these proteins, they may play crucial roles in the regulation of target genes at transcriptional level.

Sequence conservation within the MYB domain of StR2R3-MYB proteins

The R2R3-MYB proteins share significant sequence conservation within the MYB domain regions. To investigate the homologous domain sequence features into the potato R2R3-MYB domains, multiple alignments (ClustalX 2.1) were performed using the amino acid sequences of R2 and R3 repeats and sequence logos were produced to analyze the level of conservation of R2 and R3 repeats in the R2R3MYB proteins within each residue position (Fig. 3). The results revealed that five and four conserved amino acid residues were identical among all the members detected in the R2 and R3 MYB repeat regions, respectively (Fig. 3 and Additional files 4: Table S4).

Three regularly spaced tryptophan (Trp., W) residues were contained in typical MYB proteins, which play significant roles by interacting with specific DNA sequences [10]. For 106 out of the 108 potato R2R3MYB proteins, the R2 repeat sequences contained three Trp residues (Fig. 3), whereas two R2R3-MYB TFs (PG0026758 and PG0002828) only contained last two Trp residues, the first Trp was replaced by phenylalanine (Phe., F) (Fig. 3 and Additional files 4: Table S4). 
In R2 repeat, insertion of a glycine (Gly., G) residue was observed between Gly-24 and asparagine (Asn., N)-25 in five R2R3-MYB genes, an aspartic acid (Asp., D) residue in PG0002828 and PG0026758, an Asn residue and a threonine (Thr., T) residue insertion in PG0018750 and PG0035400 at same position were also observed (Additional files 4: Table S4). In addition, the insertion of the leucine (Leu., L) residue between the second and third helices of R2 repeat was observed in 90 StR2R3MYB proteins, which was an important step for the origin for plant-specific R2R3 MYB proteins [68], whereas a proline (Pro., P) residue was inserted at same position in PG0015087, furthermore, a 22-residue insertion (SFLFLLDLYSQSEFRARLIVWL) was observed between the second and third helices of R2 repeat in PG0001325.

In the R3 repeats, the first Trp residue was generally replaced by Phe or isoleucine (lle., I) (Fig. 3). The second Trp residue was conserved in all the members, the third Trp residue was conserved in most of the members, except five and four R2R3-MYB members whose third Trp residue was replaced by Phe and tyrosine (Tyr., Y), respectively. Only one valine (Val., V) residue insertion was observed between Gly-4 and Lys-5 in PG0025720 (Additional files 4: Table S4).

In addition to the highly conserved Trp residues, glutamic acid (Glu., E)-10, Asp-11 in the R2 repeat, Glu-10, Gly-22, arginine (Arg., R)-35, Asn-38, lysine (Lys., K)-41, Asn-42 in the R3 repeat were also complete conserved, Leu-50 conserved in the linker region of 107 StR2R3-MYB TFs, except the Leu was replaced by Arg in PG0034577. The result showed that R3 repeat was more conserved than the R2 repeat, furthermore, the third helix of each repeat in the MYB domain was the most conserved and the first part of the HTH domain in each repeat was less conserved among the 108 StR2R3-MYB proteins.

Chromosomal location and gene duplication of potato StR2R3-MYB genes

Genome chromosomal location analyses revealed that potato $R 2 R 3-M Y B$ genes were distributed throughout all 12 chromosomes, but the distribution appeared to be uneven (Fig. 4). A total of 105 $R 2 R 3 M Y B$ genes were mapped on 12 chromosomes, whereas three R2R3-MYB genes ( $P G 0046667$, $P G 0025720, P G 0042799)$ were remained unmapped. Chr05 encompassed the largest number of 13 $R 2 R 3-M Y B$ genes, followed by Chr06 with 12 R2R3-MYB genes. Relatively high densities of R2R3-MYBS were observed in some chromosomal regions, including the top and bottom of chromosomes $4,5,10$, and 12; the bottom of chromosomes 2, 3, 6 and 7, by contrast, most of central chromosomal regions lacked StR2R3-MYBs (Fig. 4). The pattern is similar with the genome chromosomal location of SIMYB [18].

Gene duplication has long been recognized as the origin of multigene families, and has been proved to be a prominent feature of plant genome evolution. To investigate the gene duplication events in potato, tandem duplications and segmental duplications were identified by BLASTP and MCScanX method. Among the StR2R3-MYB genes, 6 pairs of tandemly duplicated genes were identified, of which, PG0017525 was tandemly duplicated with a MYB-related gene PG0017526 (Fig. 4 and Additional files 5: Table S5). Meanwhile, 29 segmental (26.9\%) duplication pairs were found between StR2R3-MYB genes (Fig. 5 and Additional files 5: Table S5) with two exception (PG0027157 and PG0027575) were duplicated with MYB-related genes PG0004610 and PG0008340, respectively. Most segmentally duplicated MYBS 
are located in collinear regions on chr2, 3, 5 and 6. (Fig. 5), and most of these segmentally duplicated genes were belonged to $S 1,2,9,11,18$ and 20 (Additional files 5: Table S5), suggesting the rapid expansion of $R 2 R 3-M Y B$ genes in these function-specific groups in potato might be strongly linked to adaptation strategies in response to challenging environments.

To further explore the potential evolutionary processes of StR2R3-MYB gene family, two comparative syntenies of potato with Arabidopsis thaliana and Triticum aestivum (wheat), which were belonged to dicotyledon and monocotyledon respectively (Fig. 6A and 6B) were constructed. The results showed that 37 orthologs between potato and Arabidopsis, 13 orthologs between potato and wheat were identified, respectively (Additional files 6: Table S6).

The substitution rate $(\mathrm{Ka} / \mathrm{Ks})$ was an effective index to determine the positive selection pressure after duplication, $\mathrm{Ka} / \mathrm{Ks}<1$ means purifying selection, $\mathrm{Ka} / \mathrm{Ks}=1$ stands for neutral selection, while $\mathrm{Ka} / \mathrm{Ks}>1$ signifies positive selection [69]. Thus, the $\mathrm{Ka}, \mathrm{Ks}$ and $\mathrm{Ka} / \mathrm{Ks}$ of each gene pair were calculated and results showed that all the segmentally and tandemly duplicated $M Y B$ gene pairs had $\mathrm{Ka} / \mathrm{Ks}$ values of less than 1, except one pair $P G 0005918$ and $P G 0022689$ with $\mathrm{Ka} / \mathrm{Ks}=1.01$, implying that most of these genes had evolved under the effect of purifying selection (Additional files 5: Table S5 and Additional files 6: Table S6), the average $\mathrm{Ka} / \mathrm{Ks}$ value of tandem duplication genes (0.31) is a little lower than that of segmental duplication genes (0.36) (Fig. 6E), and the Ka/Ks values of gene pairs between potato and Arabidopsis, potato and wheat orthologs were 0.3315 and 0.3722 , respectively (Additional files 6: Table S6).

Expression profiles of StR2R3-MYB genes in different tissues

To understand the tissue-specific expression patterns of the StR2R3-MYB genes, the transcript abundance of 13 different tissues (leaves, roots, shoots, callus, tubers, sepals, stamens, stolons, flowers, petioles, petals, carpels and fruit) of DM potato was analyzed by using transcriptome data downloaded from the PGSC. The results showed that a total of 7.4\% StR2R3-MYB genes (8/108) were highly expressed in all tissues (Additional files 7: Fig. S1), while 23.89\% (43/108) StR2R3-MYB genes showed low expression with FPKM less than 2, of which, 7 genes showed no expression in all 13 tissues, some other genes displayed a tissue-specific expression pattern, such as 7 genes in leaf, 13 genes in root, 20 genes in shoot, 12 genes in tuber, 18 genes in stolon, and 25 genes in flower showed the higher transcript abundances with an FPKM > 5 (Additional files 7: Fig. S1 and Additional files 8: Table S7).

Expression profiles of StR2R3-MYB genes in pigmented potato cultivars

To investigate the roles of these StR2R3-MYB TFs that might be involved in anthocyanin biosynthesis, expression profiles of StR2R3-MYB genes in different tuber tissues (skin and flesh) of tetraploid pigmented potato cultivars were examined. StAN1, StMYBA1 and StMYB113 were found to influence anthocyanin biosynthesis in tobacco by transient assays [33]. in PGSC database, the best blast hits for StAN1, StMYBA1 and StMYB113 were PGS0013965, PG0013966 and PG0019217, respectively, PG0013965 and PG0013966 contained single MYB domain, while MYB domain was not found in PG0019217, their expression was also analyzed in this RNA-seq dataset. The results showed that in 
potato tuber skin, 27 StR2R3-MYB genes showed no expression, expression of 28 StR2R3-MYB genes was very low with an FPKM value less than 1. Compared with white skin of white cultivar XD, seven StR2R3MYB genes, homologous to AtMYB102 (S11), AtMYB69 (S21), AtMYB13 (S2), AtMYB74 (S11), AtMYB93 (S24) and $A t M Y B 58$ (S3), showed higher expression in pigmented skin of HM and LT, on the contrary, six StR2R3-MYB genes, homologous to AtMYB15(S2), AtMYB38 (S14), AtMYB36 (S14), AtMYB20 and AtMYB37(S14), were only highly expressed in white skin. The $P G 0013965$ showed higher expression in pigmented skin than in white skin, while the FPKM was less than 5, whereas the expression of $P G 0013966$ was higher in white skin with an FPKM $=5$, the $P G 0019217$ showed no expression (Fig. 7A and Additional files 9: Table S8).

In tuber flesh, 38 StR2R3-MYB genes were not expressed and the expression of 39 StR2R3-MYBs was lower than 1 . The $P G 0013965$ was highly expressed in purple and red flesh, while the PG0013966 and $P G 0019217$ showed lower expression in tuber flesh. In addition to $P G 0013965$, six genes, which are homologous to AtMYB48, AtMYB3(S4), AtMYB36(S14) and AtMYB79, showed higher expression in pigmented flesh, while two genes, homologous to AtMYB27 and AtMYB15(S2), showed higher expression in white flesh (Fig. 7B and Additional files 9: Table S8).

Furthermore, an interaction network of StR2R3MYB proteins, which showed expression with an FPKM value more than 1 in skin and flesh, was built using STRING software. The results showed that eight StR2R3-MYB TFs can directly interact with StAN1 with combined score $>400$, of which, two MYBs (PG0007325 and PG0018113) were homologous to AtMYB67, two MYBs (PG0024822 and PG0018427) belonged to S21, two MYBs (PG0003316 and PG0024983) belonged to S22, one MYB (PG0027190) belonged to S23 and one MYB (PG0011243) was homologous to AtMYB72. Interestingly, the two MYBs (PG003316 and PG0024983), which belonged to S22, were also interacted with StMYBA1 (Fig. 7C), suggesting the two MYBs might play important roles in anthocyanin biosynthesis. Of these MYB TFs, PG0007325 showed higher expression in pigmented skin, PG0018113 showed higher expression in purple flesh and pigmented skin, PG0024822 and PG0018427 in S21 were higher expressed in white skin, $P G 0003316$ and $P G 0024983$ in $S 22$ were all highly expressed in all tissues, $P G 0027190$ was up-regulated in pigmented flesh and $P G 0011243$ was higher expressed in pigmented skin.

Expression profiles of StR2R3-MYB genes under abiotic stress

To understand the StR2R3-MYB genes in response to abiotic stresses, expression profiles of StR2R3-MYB genes under abiotic stresses were also examined by using transcriptome data downloaded from the PGSC. The results showed that 55, 57 and 53 genes were differentially expressed with an FPKM $>1$ under salt, mannitol and heat treatment, respectively (Additional files 10: Fig. S2), of which, 47, 45 and 35 genes were up-regulated, respectively. 13 genes were responded to three stress treatments with an FPKM > 1 and $|\log 2(\mathrm{FC})|>1$, of which three genes (PG0013405, PG0017223, PG0016292) belonged to A20 (S2), two genes ( $P G 0026758$ and $P G 0002828)$ belonged to A1, two genes ( $P G 0006930$ and $P G 0019535$ ) belong to $A 13$ (S1), the other genes belonged to $A 9, A 10$ (S20), A14, (S14), A18 (S24), A26 (S11), 
respectively. 25 genes were responded to at least two stress treatments with an FPKM > 1 and |log2(FC)| $>1$ (Additional files 11: Table S9).

To further investigate the StR2R3-MYB genes might be in response to drought stress, one tetraploid drought-sensitive cultivar "Atlantic" (A) and one tetraploid drought-tolerant cultivar "Qingshu No.9" (Q) were subject to drought stress. The RNA-seq data showed that 42 StR2R3-MYB genes were up- or downregulated in response to drought stress, of which, 15 genes were highly expressed in $Q$ with an FPKM > 5 and $|\log 2(\mathrm{FC})|>1$ at flower-falling stage (Fig. 8 and Additional files 12: Table S10). There are not much differences of these genes in $A$ and $Q$ at early flowering stage, then these genes were up-regulated in $Q$ at full-blooming stage and highly up-regulated in $\mathrm{Q}$ at flower-falling stage under drought stress, while four genes were down-regulated in $Q$ beginning from early flowering stage until flower-falling stage, these genes might be involved in drought stress, which is worth further investigating.

We also analyzed expression patterns of 12 selected StR2R3-MYB genes from different subfamilies by quantitative real-time PCR (qPCR), which showed relatively higher expression by RNA-seq related to anthocyanin biosynthesis and drought stress to further confirm the reliability of RNA-seq database. The results showed that the expression pattern determined by QPCR and FPKM is consistent, as shown in Figure 9,

PG0013965 (StAN1) was up-regulated in pigmented tissues, the expression in pigmented skin was lower than that in pigmented flesh, while it was not expressed in A and Q under drought stress. PG0017223 is exclusively highly expressed in white skin, whereas $P G 0015536$ and $P G 0030548$ were only up-regulated in pigmented flesh, PG0009033 was higher expressed in Q during three stages, PG1026177, PG2026177, $P G 0024983$ and $P G 0013405$ were highly expressed in Q at flower-falling stage, two genes $P G 0015536$ and PG0030548, showed opposite expression pattern with higher expression in A at flower-falling stage. Although the relative expression of the selected genes varied between RNA-Seq dataset and qPCR analysis, a high correlation $\left(R^{2}=0.8394\right)$ described by a simple liner regression equation $y=0.8762 x+$ 0.0332 , suggests good consistency between the two analysis methods.

Identification of EAR motif-containing StR2R3-MYB proteins in potato

The Ethylene-responsive element binding-factor-associated amphiphilic repression (EAR) motif, defined by the consensus sequence patterns of either $L x L x L$ or DLNxxP is the most dominant transcriptional repression motif identified in plants. In our study, 20 members of StR2R3-MYB family have been identified containing at least one LxLxL type of EAR motif, and one StR2R3-MYB protein contained DLNxxP (Table 1), these StR2R3-MYBs were belonged to $S 2, S 4, S 9, S 18, S 20, S 22$, and two StR2R3-MYBs were homologous to AtMYB41 and AtMYB48, PG0020071 (AtMYB15-like) have two LxLxL motifs. The 22 EAR motif sites were mostly found in the C-terminal region (10 out of 22) and in the N-terminal (9 out of 22), and at lower frequency in the middle (3 out of 22) regions (Table 1).

The core EAR motif sites comprising nine amino acids were analyzed by MEME website, among the 21 LxLxLx motifs, positions 1, 2, 4 and 9 were more frequently occupied by Gln, Leu, lle and His residues, Glu 
and Ser, His and Pro are more abundant in position 6 and 8, respectively (Fig. 10A). Subsequently, the protein interactions of EAR motif-containing StR2R3-MYB TFs with other potato proteins were examined by using STRING software with combined score $>400$ (Fig. 10B and Additional files 13: Table S11). The results showed that the 21 EAR motif-containing StR2R3-MYB proteins were involved in at least nine interaction possibilities, the StR2R3-MYB proteins, belonged to the same subfamilies, appeared to have similar functions by regulating common target genes (Fig. 10).

Furthermore, GO assignments were used to predict the functions of the EAR motif-containing StR2R3MYB proteins by classifying them into three independent ontologies in terms of biological process (BP), molecular function (MF) and cellular components (CC). As shown in Additional files 14: Table S12, the functions of these proteins were related to biological process (11 out of $21,52.4 \%$ ) including biological regulation (8), cellular process (8), metabolic process (8), response to stimulus (11) and developmental process (5) etc.; for molecular function (21 out of $21,100 \%$ ), nucleic acid binding (21) was the most represented GO term, followed by calmodulin binding (1); for cellular component (6 out of 21, 28.6\%), major categories were cell (6) and cell part (6).

\section{Discussion}

The MYB family is one of the largest transcription factors families, which have been identified to be involved in various plant physiological and biochemical processes. R2R3-MYBs are the predominant form found in higher plants, they play important roles in the primary and secondary metabolism, developmental processes and responses to biotic and abiotic stresses [5]. In the present work, we performed a genome-wide investigation of the $R 2 R 3-M Y B$ gene family in potato. A total of $108 R 2 R 3-$ $M Y B s$ were identified, most of which remain to be functionally characterized. Subsequently, a phylogenetic tree was constructed to evaluate the evolutionary relationships of $R 2 R 3-M Y B$ genes between potato and Arabidopsis to identify the evolution and possible functions of StR2R3-MYBs. Detailed analyses including gene structures, conserved motif composition, chromosomal location and gene duplication events were performed to further identify the characteristics of these StR2R3-MYB genes. In addition, the expression profiles of StR2R3-MYB genes in different tissues, in skin and flesh of pigmented potato cultivars as well as in response to different abiotic stresses, especially drought stress were analyzed. Finally, 21 EAR motif-containing StR2R3-MYB proteins were identified and the interaction network of these proteins with their interacting proteins were analyzed to investigate the transcriptional regulatory mechanisms of potato StR2R3-MYB gene family.

Phylogenetic analysis of the potato R2R3-MYB genes

Generally, paralogous and orthologous functional relationships can be confirmed by knowing the functions of certain members, it is likely that members within a subgroup may have common evolutionary origins and a conserved function. According to the phylogenetic comparative analysis, most StR2R3-MYBs are clustered with orthologs of AtR2R3-MYBs in different subgroups (Fig.1 and Fig. 2). For example, A17 consists of PG0009033, PG0004371 and PG0007304, which were clustered with AtMYB11, 
AtMYB12, and AtMYB111 (S7), implicating in the control of flavonoid accumulation [70-72], A16 comprises four members clustered with AtMYB123/TT2 (S5), which controls the biosynthesis of proanthocyanidins (PAs) in the seed coat of Arabidopsis [73].

Duplication contributed to the StR2R3-MYB gene expansion

Gene duplication contributes significantly to the expansion of MYB genes in the plant kingdom, which lead to the diversification and evolution of genes. Our results showed that $6(5.6 \%)$ and $29(26.9 \%)$ StR2R3-MYB genes were identified as tandem duplication and segmental duplication, respectively, indicated that segmental duplication event was a major cause of expansion of StR2R3-MYB genes.

Most StR2R3-MYB genes were tandemly and segmentally duplicated within one cluster to expand their subfamilies, the duplication event was mostly occurred in the genes belong to S1, S2, S18 and S20 (Additional files 5: Table S5), exceptionally, one StR2R3-MYB gene $P G 0017525$ was tandemly duplicated with MYB-related gene (PG0017526), two StR2R3-MYB genes (PG0027157 and PG0027575) were segmentally duplicated with two MYB-related genes ( $P G 0004610$ and PG0008340).

Some evolving members may have lost their original function(s) and/or acquiring new function(s) to enhance the adaptability of plants or become pseudogenes as a result of duplication event [74]. In our work, we found that some pairs of StR2R3-MYB genes displayed a different expression level in different tissues and under abiotic stresses, whereas some pairs of StR2R3-MYB genes were presented similar expression pattern. For example, in one pair, PG1026177 and PG2026177 were all highly expressed in drought-tolerant cultivar Q at flower-falling stage under drought stress, one gene PG0026176, which tandemly duplicated with $P G 2026177$, was not expressed under drought stress.

Identification of the StR2R3-MYB TFs might be involved in anthocyanin biosynthesis

In Arabidopsis, 12 representative members of MYB family proteins, such as MYB75, MYB90, MYB113, MYB114, MYB3, MYB4, MYB7, MYB11, MYB12, MYB111, MYB32, and MYB60 were involved in controlling some major phenylpropanoid biosynthesis genes $[2,5,72]$. In our work, four members (A18) were clustered with AtMYB3, AtMYB4, AtMYB7 (S4), three members (A17) were clustered with AtMYB11, AtMYB12, and AtMYB111 (S7), one MYB PG0028125 was clustered with AtMYB60. In the RNA-seq database of pigmented skin and flesh, three members of $A 17$ were not expressed in either white or pigmented skin, while $P G 0009033$ was up-regulated in pigmented flesh, in A18, PG0030548 was upregulated in pigmented skin and flesh, while last three members showed no difference between white and pigmented tissues. PG0028125, which is homologous to AtMYB60, showed little expression in white and pigmented tissues.

Our previous work showed that in transient assays, StMYB44 represses anthocyanin accumulation in leaves of $N$. tabacum and $N$. benthamiana by directly suppressing the activity of the dihydroflavonol reductase (DFR) promoter. StMYB44-1 (PG0003316) showed stronger repressive capacity than StMYB442 (PG0007994) with both predicted proteins containing the repression-associated EAR motif with some 
variation (Liu et al. 2018). In our RNA-seq dataset, the two StMYB44s were all expressed in white and pigmented skin and flesh, as well as another AtMYB44-like gene PG0024983; one gene (PG0030548) belonged to $S 4$, which was reported to repress flavonoid/anthocyanin accumulation, was highly expressed in pigmented flesh. It's also reported that the MdMYB15L, homologous to AtMYB15, might act upon MBW complexes to repress anthocyanin accumulation by interacting with MdbHLH33 [75], in our dataset, two genes PG0017223 and PG0016292, homologous to AtMYB15(S2), were highly expressed in white skin. The results suggest that hierarchical interactions among R2R3-MYB regulators are complicated and requires further investigation, which was revealed by Albert et al. (2014) [76] that the network of transcriptional activators and repressors involved in anthocyanin accumulation was conserved in eudicots and by Zhou et al. (2018) [77] that activator-type R2R3-MYB genes induce a repressor-type R2R3-MYB gene to balance anthocyanin and proanthocyanidin accumulation.

The StR2R3-MYB TFs respond to abiotic stresses

The MYB TFs play essential roles in the regulation of gene expression to cope with environmental stresses [78, 79]. 67 StR2R3-MYBs were differentially expressed under salt, mannitol and heat stresses, indicating that they were major factors involving in crosstalk among different signal transduction pathways in response to abiotic stresses. However, several StR2R3-MYB genes appeared to take part in respond to only one stress stimulus, suggest that there are different signaling pathways related to the response to abiotic stress treatment. In addition, some genes showed opposite expression profiles under different stresses, implying the complicated signaling transduction pathways in response to abiotic stresses. We further analyzed StR2R3-MYB TFs might be involved in drought stress based on RNA-seq data and compared with the data treated with mannitol downloaded from PGSC, the results showed that 15 genes were highly expressed in drought-tolerant cultivar $Q$ with an FPKM $>5$ and $|\log 2(\mathrm{FC})|>1$ at flower-falling stage (Fig. 8 and Additional files 12: Table S10), of which, 10 genes showed the same expression pattern in two RNA-seq datasets, while two genes (PG0015536 and PG0022689) showed opposite expression pattern, two genes (PG2026177 and PG1026177) in A22 and one gene (PG0005848) in A16 (S5) were highly expressed in Q, while showed lower expression in mannitol-treated RNA dataset. Among these 15 genes, only two genes (PG0030548 and PG0031317), all belonged to S4, were highly expressed in A, while other genes were all highly expressed in Q. It's reported that AtMYB44/AtMYBR1 (S22) regulates ABA-mediated stomatal closure in response to abiotic stresses and three other members (AtMYB70, AtMYB73 and AtMYB77) in this subgroup are likely to be associated with stress responses $[80,81]$. In our present work, one member PG0024983 in S22 was up-regulated under salt and mannitol stresses, and highly expressed in Q at flowering-falling stage as well, the other member $P G 0003316$ in S22 was highly expressed under mannitol treatment, also up-regulated in Q, suggesting the two StR2R3$M Y B$ genes are likely to be associated with drought response. AtMYB96 mediated the abscisic acid (ABA) signal network that confers abiotic stress tolerance [82], in our dataset, PG0033043 and PG0019535, homologous to AtMYB96, were all up-regulated in DM potato under salt and mannitol stresses, and PG0033043 was up-regulated in Q as well. The results revealed that these identified StR2R3-MYB TFs were responded to drought stress, and was worth further investigating. Our genome-wide analysis and 
expression profiles of StR2R3-MYB TFs in response to various stresses, especially drought stress, provide a foundation for their functional characterization with stress tolerance.

The interaction network of EAR motif-containing StR2R3-MYB proteins

A transcription factor can act as an activator or repressor in the transcriptional regulation [83]. The EAR motif is the most dominant transcriptional repression motif identified in plants. These R2R3 MYB repressors include AtMYB3/4/6 in Arabidopsis [84], MdMYB16/17/111 in apple [85], FaMYB1 in strawberry [86] and PhMYB4 and PhMYB17 in petunia [87]. Our previous work also suggested that StMYB44-1 (PG0003316) exerted its strong repressive ability through the presence of a complete EAR motif $(\operatorname{LxLXXX)}$ in the C-terminal region. The sequence of this motif is less conserved in StMYB44-2, with a substitution of the final $L$ with $P(L x L x P x)$ which could account for its weaker repression [88]. In this work, 20 members of StR2R3-MYB family have been identified containing at least one LxLXL type of EAR motif, and one StR2R3-MYB contained DLNxxP, and a protein interaction network of EAR motif-containing StR2R3-MYB TFs with other potato proteins showed that the 21 EAR motif-containing StR2R3-MYB proteins were involved in at least nine interaction possibilities, The investigation of EAR motif-containing proteins will provide new insight into the link between complicated gene regulation system.

\section{Conclusions}

A comprehensive analysis of R2R3-MYB gene family in potato was carried out in the present study. A total of 108 StR2R3-MYBs were identified and phylogenetically divided into 28 distinct subfamilies, as supported by highly conserved gene structures and motifs. They were unevenly distributed among 12 chromosomes in potato. Collinearity analysis showed that the segmental duplication events played a crucial role in the expansion of StR2R3-MYB gene family. Synteny analysis indicated that 37 and 13 StR2R3-MYB genes were orthologous to Arabidopsis and wheat, respectively, and these gene pairs have evolved under strong purifying selection. Moreover, we performed a comprehensive expression analysis of StR2R3-MYB genes in different tissues and under abiotic stresses in doubled monoploid (DM) potato, as well as in white and pigmented potato cultivars and in drought-sensitive and -tolerant cultivars of tetraploid potato using RNA-seq data to identify StR2R3-MYB genes closely associated with spatial distribution, anthocyanin biosynthesis and multiple stresses. In addition, 21 StR2R3-MYB genes containing EAR motif were obtained and an interaction network between these EAR motif-containing R2R3-MYBs with other potato genes were constructed. Gene Ontology (GO) analysis of the 21 EAR motifcontaining StR2R3-MYB proteins was also performed to further investigate their functions. The findings should inform the characterization of StR2R3-MYB superfamily and provide valuable information for further functional elucidation of these genes in potato.

\section{Abbreviations}

DM: doubled monoploid GO: GeneOntology 
HMM: hidden Markov model Ka: non-synonymous substitution rate

Ks: synonymous substitution rate $\mathrm{NJ}$ : Neighbor-Joining

PG: PGSC0003DMG40 qRT-PCR: quantitative real-time PCR

TF: transcription factor

\section{Declarations}

Acknowledgements

Not applicable.

Funding

The Research Program Sponsored by the Special Funds for Discipline Construction of Gansu Agricultural University (GSAU-XKJS-2018-218), National Natural Science Foundation of China (31601356 and 31860398), Gansu Science Foundation for Distinguished Young Scholars (17JR5RA138), China Agriculture Potato Research System (CARS-09-P14) and Gansu Provincial Key Laboratory of Aridland Crop Science, Gansu Agricultural University (No. GSCS-2017-2).

Availability of data and materials

The raw data of the transcriptome analysis used in this study was submitted to the Sequence Read Archive (SRA) at NCBI under Project ID PRJNA528685 and PRJNA529980, and the expression data was also available Potato Genome Sequencing Consortium (PGSC, http://solanaceae.plantbiology.msu.edu/pgsc_download.shtml).

Authors' contributions

$\mathrm{KW}, \mathrm{ACA}, \mathrm{JZ}$ and $\mathrm{YL}$ managed this project; $\mathrm{YL}, \mathrm{KW}$ and $\mathrm{YL}$ analyzed the sequencing data; $\mathrm{YL}$ and $\mathrm{ZL}$ performed the experiments; $\mathrm{YL}, \mathrm{KW}$ and $\mathrm{YL}$ wrote the manuscript; All authors read and approved the final manuscript for publication.

Ethics approval and consent to participate

Not applicable.

Consent for publication

Not applicable.

Competing interests

The authors declare that they have no competing interests. 


\section{References}

1. Antje F, Katja M, Braun EL, Erich G: Evolutionary and comparative analysis of MYB and bHLH plant transcription factors. Plant J 2011, 66(1):94-116.

2. Stracke R, Werber M, Weisshaar B: The R2R3-MYB gene family in Arabidopsis thaliana. Curr Opin Plant Biol 2001, 4(5):447-456.

3. Pabo CO, Sauer RT: Transcription factors: structural families and principles of DNA recognition. Annu Rev Biochem 1992, 61:1053-1095.

4. Riechmann JL, Ratcliffe OJ: A genomic perspective on plant transcription factors. Curr Opin Plant Biol 2000, 3(5):423-434.

5. Dubos C, Stracke R, Grotewold E, Weisshaar B, Martin C, Lepiniec Lc: MYB transcription factors in Arabidopsis. Trends Plant Sci 2010, 15(10):573-581.

6. Rosinski JA, Atchley WR: Molecular Evolution of the Myb Family of Transcription Factors: Evidence for Polyphyletic Origin. J Mol Evol 1998, 46(1):74-83.

7. Du H, Wang YB, Xie Y, Liang Z, Jiang SJ, Zhang SS, Huang YB, Tang YX: Genome-wide identification and evolutionary and expression analyses of MYB-related genes in land plants. DNA Res 2013, 20(5):437448.

8. Kanei-Ishii C, Sarai A, Sawazaki T, Nakagoshi H, He DN, Ogata K, Nishimura Y, Ishii S: The tryptophan cluster: a hypothetical structure of the DNA-binding domain of the myb protooncogene product. $J$ Biol Chem 1990, 265(32):19990-19995.

9. Ogata K, Kanei-Ishii C, Sasaki M, Hatanaka H, Nagadoi A, Enari M, Nakamura H, Nishimura Y, Ishii S, Sarai A: The cavity in the hydrophobic core of Myb DNA-binding domain is reserved for DNA recognition and trans-activation. Nat Struct Bio/ 1996, 3(2):178-187.

10. Ogata K, Nishimura Y: Specific DNA recognition by Myb protein. Tanpakushitsu Kakusan Koso 1995, 40(10):1592-1597.

11. Ogata K: Structure and dynamics of the transcription factor, Myb, in DNA-sequence recognition. Seikagaku 1998, 70(10):1233.

12. Martin C, Paz-Ares J: MYB transcription factors in plants. Trends Genet 1997, 13(2):67-73.

13. Klempnauer KH, Gonda TJ, Bishop JM: Nucleotide sequence of the retroviral leukemia gene $v-m y b$ and its cellular progenitor $c$-myb: the architecture of a transduced oncogene. Cell 1982, 31(2 Pt 1):453463.

14. Weston K: Myb proteins in life, death and differentiation. Curr Opin Genet Dev 1998, 8(1):76-81. 
15. Kranz H, Scholz K, Weisshaar B: $c-M Y B$ oncogene-like genes encoding three MYB repeats occur in all major plant lineages. Plant $J$ 2000, 21(2):231-235.

16. Paz-Ares J, Ghosal D, Wienand U, Peterson P, Saedler $\mathrm{H}$ : The regulatory $c 1$ locus of Zea mays encodes a protein with homology to myb proto-oncogene products and with structural similarities to transcriptional activators. EMBO J 1987, 6(12):3553.

17. Hajiebrahimi A, Owji H, Hemmati S: Genome-wide identification, functional prediction, and evolutionary analysis of the R2R3-MYB superfamily in Brassica napus. Genome 2017, 60(10):797-814.

18. Li ZJ, Peng RH, Tian YS, Han HJ, Xu J, Yao QH: Genome-wide identification and analysis of the MYB transcription factor superfamily in Solanum lycopersicum. Plant Cell Physiol 2016, 57(8):1657-1677.

19. Wilkins O, Nahal H, Foong J, Provart NJ, Campbell MM: Expansion and diversification of the Populus R2R3-MYB family of transcription factors. Plant Physiol 2009, 149(2):981-993.

20. Du H, Feng BR, Yang SS, Huang YB, Tang YX: The R2R3-MYB transcription factor gene family in Maize. PLOS ONE 2012, 7(6):12.

21. Du H, Yang SS, Liang Z, Feng BR, Liu L, Huang YB, Tang YX: Genome-wide analysis of the MYB transcription factor superfamily in soybean. BMC Plant Biol 2012, 12:22.

22. Cao ZH, Zhang SZ, Wang RK, Zhang RF, Hao YJ: Genome wide analysis of the apple MYB transcription factor family allows the identification of MdoMYB121 gene confering abiotic stress tolerance in plants. PLOS ONE 2013, 8(7):13.

23. Liu CY, Xie T, Chen CJ, Luan AP, Long JM, Li CH, Ding YQ, He YH: Genome-wide organization and expression profiling of the R2R3-MYB transcription factor family in pineapple (Ananas comosus). BMC Genomics 2017, 18:16.

24. Ambawat S, Sharma P, Yadav NR, Yadav RC: MYB transcription factor genes as regulators for plant responses: an overview. Physiol Mol Biol Plants 2013, 19(3):307-321.

25. Feller A, Machemer K, Braun EL, Grotewold E: Evolutionary and comparative analysis of MYB and bHLH plant transcription factors. Plant $J$ 2011, 66(1):94-116.

26. Jin $\mathrm{H}$, Martin C: Multifunctionality and diversity within the plant MYB-gene family. Plant Mol Biol 1999, 41(5):577-585.

27. Gonzalez A, Zhao M, Leavitt JM, Lloyd AM: Regulation of the anthocyanin biosynthetic pathway by the TTG1/bHLH/Myb transcriptional complex in Arabidopsis seedlings. Plant J 2008, 53(5):814-827.

28. Stracke R, Ishihara H, Huep G, Barsch A, Mehrtens F, Niehaus K, Weisshaar B: Differential regulation of closely related R2R3-MYB transcription factors controls flavonol accumulation in different parts of the 
Arabidopsis thaliana seedling. Plant J 2007, 50(4):660-677.

29. Espley RV, Hellens RP, Putterill J, Stevenson DE, Kutty-Amma S, Allan AC: Red colouration in apple fruit is due to the activity of the MYB transcription factor, MdMYB10. Plant $J$ 2007, 49(3):414-427.

30. Chagné D, Lin-Wang K, Espley RV, Volz RK, How NM, Rouse S, Brendolise C, Carlisle CM, Kumar S, De Silva N: An ancient duplication of apple MYB transcription factors is responsible for novel red fruit-flesh phenotypes. Plant Physiol 2013, 161(1):225-239.

31. Ballester AR, Molthoff J, De RV, Hekkert B, Orzaez D, Fernándezmoreno JP, Tripodi P, Grandillo S, Martin C, Heldens J: Biochemical and molecular analysis of pink tomatoes: deregulated expression of the gene encoding transcription factor SIMYB12 leads to pink tomato fruit color. Plant Physiol 2010, 152(1):71-84.

32. Jung CS, Griffiths HM, De Jong DM, Cheng S, Bodis M, Kim TS, De Jong WS: The potato developer (D) locus encodes an R2R3 MYB transcription factor that regulates expression of multiple anthocyanin structural genes in tuber skin. Theor Appl Genet 2009, 120(1):45-57.

33. Liu Y, Kui LW, Espley RV, Wang L, Yang H, Yu B, Andrew D, Erika VG, Wang J, Zhang J: Functional diversification of the potato R2R3 MYB anthocyanin activators AN1, MYBA1, and MYB113 and their interaction with basic helix-loop-helix cofactors. J Exp Bot 2016, 67(8):2159-2176.

34. Chen TZ, Li WJ, Hu XH, Guo JR, Liu AM, Zhang BL: A cotton MYB transcription factor, GbMYB5, is positively involved in plant adaptive response to drought stress. Plant Cell Physiol 2015, 56(5):917-929.

35. Cheng L, Li X, Huang X, Ma T, Liang Y, Ma X, Peng X, Jia J, Chen S, Chen Y: Overexpression of sheepgrass R1-MYB transcription factor LcMYB1 confers salt tolerance in transgenic Arabidopsis. Plant Physiol Biochem 2013, 70(1):252-260.

36. Shin D, Moon SJ, Han S, Kim BG, Park SR, Lee SK, Yoon HJ, Lee HE, Kwon HB, Baek D et al: Expression of StMYB1R-1, a novel potato single MYB-like domain transcription factor, increases drought tolerance. Plant Physiol 2011, 155(1):421-432.

37. Spooner DM, Karen ML, Gavin R, Robbie W, Bryan GJ: A single domestication for potato based on multilocus amplified fragment length polymorphism genotyping. Proc Natl Acad Sci U S A 2005, 102(41):14694-14699.

38. Fossen T, Andersen $\varnothing$ : Anthocyanins from tubers and shoots of the purple potato, Solanum tuberosum. J Hortic Sci Biotec 2000, 75(3):360-363.

39. Christie PJ, Alfenito MR, Walbot V: Impact of low-temperature stress on general phenylpropanoid and anthocyanin pathways: enhancement of transcript abundance and anthocyanin pigmentation in maize seedlings. Planta 1994, 194(4):541-549. 
40. André CM, Schafleitner R, Legay S, Lefèvre I, Aliaga CAA, Nomberto G, Hoffmann L, Hausman J-F, Larondelle Y, Evers D: Gene expression changes related to the production of phenolic compounds in potato tubers grown under drought stress. Phytochemistry 2009, 70(9):1107-1116.

41. Castellarin SD, Pfeiffer A, Sivilotti P, Degan M, Peterlunger E, Di Gaspero G: Transcriptional regulation of anthocyanin biosynthesis in ripening fruits of grapevine under seasonal water deficit. Plant Cell Environ 2007, 30(11):1381-1399.

42. He J, Giusti MM: Anthocyanins: natural colorants with health-promoting properties. Annu Rev Food Sci Technol 2010, 1:163-187.

43. Gregory PJ, Simmonds LP: Water relations and growth of potatoes. World Crops 1992:214-246.

44. Jefferies RA: Responses of potato genotypes to drought. I. Expansion of individual leaves and osmotic adjustment. Ann Appl Biol 2010, 122(1):93-104.

45. Schafleitner R, Gutierrez Rosales RO, Gaudin A, Alvarado Aliaga CA, Martinez GN, Tincopa Marca LR, Bolivar LA, Delgado FM, Simon R, Bonierbale M: Capturing candidate drought tolerance traits in two native Andean potato clones by transcription profiling of field grown plants under water stress. Plant Physiol Biochem 2007, 45(9):673-690.

46. Evers D, Lefevre I, Legay S, Lamoureux D, Hausman JF, Rosales ROG, Marca LRT, Hoffmann L, Bonierbale M, Schafleitner R: Identification of drought-responsive compounds in potato through a combined transcriptomic and targeted metabolite approach. J Exp Bot 2010, 61(9):2327-2343.

47. Moon SJ, Han SY, Kim DY, Yoon IS, Shin D, Byun MO, Kwon HB, Kim BG: Ectopic expression of a hot pepper bZIP-like transcription factor in potato enhances drought tolerance without decreasing tuber yield. Plant Mol Biol 2015, 89(4-5):421-431.

48. Wang L, Liu Y, Feng S, Yang J, Li D, Zhang J: Roles of Plasmalemma Aquaporin Gene StPIP1 in Enhancing Drought Tolerance in Potato. Frontiers in Plant Science 2017, 8:616.

49. D'Amelia V, Aversano R, Batelli G, Caruso I, Castellano Moreno M, Castro-Sanz AB, Chiaiese P, Fasano C, Palomba F, Carputo D: High AN1 variability and interaction with basic helix-loop-helix co-factors related to anthocyanin biosynthesis in potato leaves. Plant J 2014, 80(3):527-540.

50. Tai HH, Goyer C, Murphy AM: Potato MYB and bHLH transcription factors associated with anthocyanin intensity and common scab resistance. Botany 2013, 91(10):722-730.

51. Consortium PGS: Genome sequence and analysis of the tuber crop potato. Nature 2011, 475(7355):189-195.

52. Finn RD, Coggill P, Eberhardt RY, Eddy SR, Mistry J, Mitchell AL, Potter SC, Punta M, Qureshi M, Sangrador-Vegas A et al: The Pfam protein families database: towards a more sustainable future. Nucleic 
Acids Res 2016, 44(D1):D279-D285.

53. Yanhui C, Xiaoyuan Y, Kun H, Meihua L, Jigang L, Zhaofeng G, Zhiqiang L, Yunfei Z, Xiaoxiao W, Xiaoming Q et al: The MYB transcription factor superfamily of Arabidopsis: expression analysis and phylogenetic comparison with the rice MYB family. Plant Mol Biol 2006, 60(1):107-124.

54. Letunic I, Doerks T, Bork P: SMART: recent updates, new developments and status in 2015. Nucleic Acids Res 2015, 43(D1):D257-D260.

55. Marchler-Bauer A, Derbyshire MK, Gonzales NR, Lu SN, Chitsaz F, Geer LY, Geer RC, He J, Gwadz M, Hurwitz DI et al: CDD: NCBI's conserved domain database. Nucleic Acids Res 2015, 43(D1):D222-D226.

56. Hu B, Jin JP, Guo AY, Zhang H, Luo JC, Gao G: GSDS 2.0: an upgraded gene feature visualization server. Bioinformatics 2015, 31(8):1296-1297.

57. Bailey TL, Boden M, Buske FA, Frith M, Grant CE, Clementi L, Ren J, Li WW, Noble WS: MEME SUITE: tools for motif discovery and searching. Nucleic Acids Res 2009, 37(Web Server issue):W202-208.

58. Voorrips RE: MapChart: software for the graphical presentation of linkage maps and QTLs. J Hered 2002, 93(1):77-78.

59. Wang Y, Tang H, Debarry Jeremy D, Tan X, Li J, Wang X, Lee T-H, Jin H, Marler B, Guo H: MCScanX: a toolkit for detection and evolutionary analysis of gene synteny and collinearity. Nucleic Acids Res 2012, 40(7):e49-e49.

60. Krzywinski M, Schein Jl: Circos: an information aesthetic for comparative genomics. Genome Res 2009, 19(9):1639-1645.

61. Wang D, Zhang Y, Zhang Z, Zhu J, Yu J: KaKs_Calculator 2.0:A Toolkit Incorporating Gamma-Series Methods and Sliding Window Strategies. Genom Proteom bioinf 2010, 8(1):77-80.

62. Kumar S, Stecher G, Tamura K: MEGA7: Molecular Evolutionary Genetics Analysis version 7.0 for bigger datasets. Mol Biol Evol 2016, 33(7):1870.

63. Tang X, Zhang N, Si H, CalderÃ $\tilde{}^{3}$-Urrea A: Selection and validation of reference genes for RT-qPCR analysis in potato under abiotic stress. Plant Methods 2017, 13(1):85.

64. Chen C, Xia R, Chen H, He Y: TBtools, a Toolkit for Biologists integrating various HTS-data handling tools with a user-friendly interface. bioRxiv 2018:289660.

65. Kagale S, Rozwadowski K: EAR motif-mediated transcriptional repression in plants: An underlying mechanism for epigenetic regulation of gene expression. Epigenetics 2011, 6(2):141-146.

66. Szklarczyk D, Morris JH, Cook H, Kuhn M, Wyder S, Simonovic M, Santos A, Doncheva NT, Roth A, Bork $P$ et al: The STRING database in 2017: quality-controlled protein-protein association networks, made 
broadly accessible. Nucleic Acids Res 2017, 45(D1):D362-D368.

67. Shannon P, Markiel A, Ozier O, Baliga NS, Wang JT, Ramage D, Amin N, Schwikowski B, Ideker T: Cytoscape: a software environment for integrated models of biomolecular interaction networks. Genome Res 2003, 13(11):2498-2504.

68. Williams CE, Grotewold E: Differences between plant and animal Myb domains are fundamental for DNA binding activity, and chimeric Myb domains have novel DNA binding specificities. J Biol Chem 1997, 272(1):563-571.

69. Lynch M, ., Conery JS: The evolutionary fate and consequences of duplicate genes. Science 2000, 290(5494):1151-1155.

70. Pandey A, Misra P, Bhambhani S, Bhatia C, Trivedi PK: Expression of Arabidopsis MYB transcription factor, AtMYB111, in tobacco requires light to modulate flavonol content. Sci Rep 2014, 4(5):5018.

71. Pandey A, Misra P, Choudhary D, Yadav R, Goel R, Bhambhani S, Sanyal I, Trivedi R, Trivedi PK: AtMYB12 expression in tomato leads to large scale differential modulation in transcriptome and flavonoid content in leaf and fruit tissues. Sci Rep 2015, 5(12412):12412.

72. Stracke R, Ishihara HG, Barsch A, Mehrtens F, Niehaus K, Weisshaar B: Differential regulation of closely related R2R3-MYB transcription factors controls flavonol accumulation in different parts of the Arabidopsis thaliana seedling. Plant J 2010, 50(4):660-677.

73. Lepiniec L, Debeaujon I, J M, Baudry A, Pourcel L, Nesi N, Caboche M: Genetics and biochemistry of seed flavonoids. Annu Rev Plant Bio/ 2006, 57(1):405-430.

74. Dias AP, Braun EL, Mcmullen MD, Grotewold E: Recently duplicated maize R2R3 myb genes provide evidence for distinct mechanisms of evolutionary divergence after duplication. Plant Physio/ 2003, 131(2):610-620.

75. Xu HF, Yang GX, Zhang J, Wang YC, Zhang TL, Wang N, Jiang SH, Zhang ZY, Chen XS: Overexpression of a repressor MdMYB15L negatively regulates anthocyanin and cold tolerance in red-fleshed callus. Biochem Biophys Res Commun 2018, 500(2):405-410.

76. Albert NW, Davies KM, Lewis DH, Zhang H, Montefiori M, Brendolise C, Boase MR, Ngo H, Jameson PE, Schwinn KE: A conserved network of transcriptional activators and repressors regulates anthocyanin pigmentation in eudicots. Plant Cell 2014, 26(3):962-980.

77. Zhou H, Lin-Wang K, Wang F, Espley RV, Ren F, Zhao J, Ogutu C, He H, Jiang Q, Allan AC et al: Activator-type R2R3-MYB genes induce a repressor-type R2R3-MYB gene to balance anthocyanin and proanthocyanidin accumulation. New Phytol 2019, 221(4):1919-1934. 
78. Takashi H, Kazuo S: Research on plant abiotic stress responses in the post-genome era: past, present and future. Plant J 2010, 61(6):1041-1052.

79. Ahuja I, Vos RCHD, Bones AM, Hall RD: Plant molecular stress responses face climate change. Trends Plant Sci 2010, 15(12):664-674.

80. Jung C, Seo JS, Han SW, Koo YJ, Kim CH, Song SI, Nahm BH, Choi YD, Cheong J-J: Overexpression of AtMYB44 enhances stomatal closure to confer abiotic stress tolerance in transgenic Arabidopsis. Plant Physiol 2008, 146(2):623-635.

81. Seo JS, Sohn HB, Noh K, Jung C, An JH, Donovan CM, Somers DA, Kim DI, Jeong SC, Kim CG et al: Expression of the Arabidopsis AtMYB44 gene confers drought/salt-stress tolerance in transgenic soybean. Mol Breed 2012, 29(3):601-608.

82. Seo PJ, Xiang F, Qiao M, Park J-Y, Lee YN, Kim S-G, Lee Y-H, Park WJ, Park C-M: The MYB96 transcription factor mediates abscisic acid signaling during drought stress response in Arabidopsis. Plant Physio/ 2009, 151(1):275-289.

83. Latchman DS: Transcription factors: an overview. Int J Biochem Cell B 1997, 29(12):1305-1312.

84. Jin H, Cominelli E, Bailey P, Parr A, Mehrtens F, Jones J, Tonelli C, Weisshaar B, Martin C: Transcriptional repression by AtMYB4 controls production of UV-protecting sunscreens in Arabidopsis. EMBO J 2000, 19(22):6150-6161.

85. Lin-Wang K, Micheletti D, Palmer J, Volz R, Lozano L, Espley R, Hellens RP, Chagne D, Rowan DD, Troggio $\mathrm{M}$ et al: High temperature reduces apple fruit colour via modulation of the anthocyanin regulatory complex. Plant Cell Environ 2011, 34(7):1176-1190.

86. Aharoni A, De Vos C, Wein M, Sun Z, Greco R, Kroon A, Mol JN, O'Connell AP: The strawberry FaMYB1 transcription factor suppresses anthocyanin and flavonol accumulation in transgenic tobacco. Plant $J$ 2001, 28(3):319-332.

87. Albert NW, Lewis DH, Zhang H, Schwinn KE, Jameson PE, Davies KM: Members of an R2R3-MYB transcription factor family in Petunia are developmentally and environmentally regulated to control complex floral and vegetative pigmentation patterning. Plant $J$ 2011, 65(5):771-784.

88. Liu Y, Lin-Wang K, Espley RV, Wang L, Li Y, Liu Z, Zhou P, Zeng L, Zhang X, Zhang J et al: StMYB44 negatively regulates anthocyanin biosynthesis at high temperatures in tuber flesh of potato. $J$ Exp Bot 2019.

\section{Tables}

Table 1 potato StR2R3-MYB proteins with conserved EAR motifs 
Homologous to At Gene ID Size EAR motif

\begin{tabular}{|c|c|c|c|c|c|}
\hline & & & & sequence & Location \\
\hline \multirow[t]{2}{*}{1} & MYB15-like S2 & PG0020071 & 259 & NYLDLSLME & 220 \\
\hline & & PG0020071 & 259 & NELMLELPE & 251 \\
\hline 2 & MYB32-like S4 & PG0006176 & 255 & PDLNLELRI & 188 \\
\hline 3 & MYB4-like S4 & PG0013215 & 269 & PDLNLELRI & 189 \\
\hline 4 & MYB3-like S4 & PG0030548 & 179 & LLDLNSLP* & 173 \\
\hline 5 & MYB17-like S9 & PG0000027 & 310 & SALQLLLDF & 278 \\
\hline 6 & MYB17-like S9 & PG0021654 & 320 & SALQLLLDF & 274 \\
\hline 7 & MYB101-like S18 & PG0028949 & 485 & KGLPLTLPS & 200 \\
\hline 8 & MYB101-like S18 & PG0013897 & 477 & VSLSLTLAS & 237 \\
\hline 9 & MYB108-like S20 & PG0008761 & 298 & QLLILELHS & 89 \\
\hline 10 & MYB62-like S20 & PG0014550 & 274 & QLLILELHS & 84 \\
\hline 11 & MYB108-like S20 & PG0027157 & 325 & QLLILELHS & 104 \\
\hline 12 & MYB108-like S20 & PG2004611 & 235 & QLLILQLHS & 68 \\
\hline 13 & MYB108-like S20 & PG1004611 & 296 & QLLILQLHS & 100 \\
\hline 14 & MYB108-like S20 & PG0004612 & 231 & QLLILQLHF & 68 \\
\hline 15 & MYB62-like S20 & PG0005641 & 279 & QLLILELHS & 88 \\
\hline 16 & MYB70-like S22 & PG0044858 & 305 & THLSLSLPG & 227 \\
\hline 17 & MYB44-like S22 & PG0003316 & 321 & TSLCLSLPG & 206 \\
\hline 18 & AtMYB73-like S22 & PG0024983 & 372 & TSLSLSLPG & $205-209$ \\
\hline 19 & MYB41-like & PG0020012 & 244 & FWLELYLAA & 232 \\
\hline 20 & MYB15-like & PG0015087 & 244 & DELILNLHA & 77 \\
\hline 21 & MYB48-like & PG0015536 & 219 & ERLVLELHS & 72 \\
\hline
\end{tabular}

PG represents PGSC0003DMG40.

Figures 


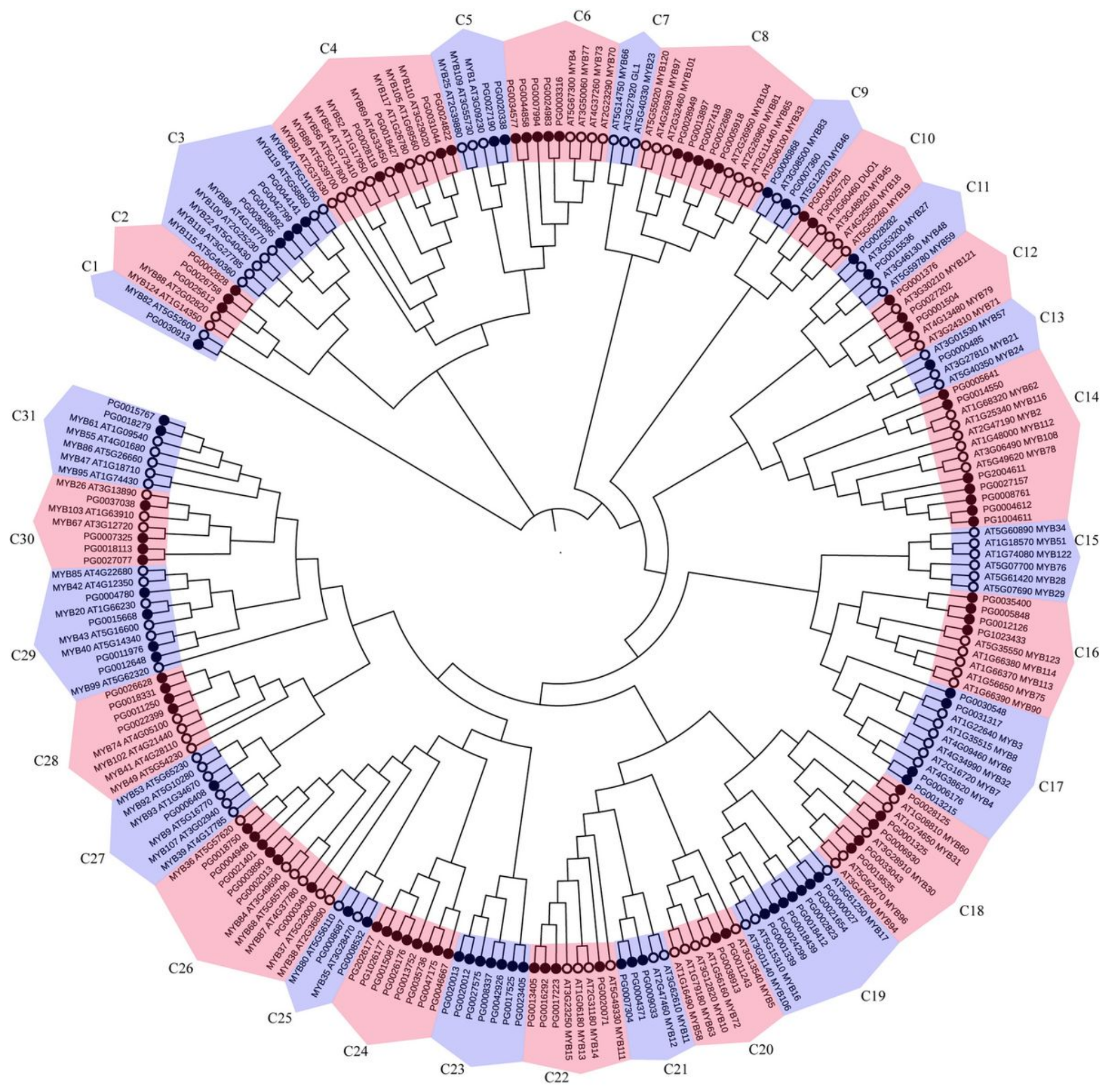

Figure 1

Phylogenetic relationships of potato and Arabidopsis R2R3-MYB proteins. The complete amino acid sequences of the 126 Arabidopsis, and 108 potato R2R3-MYB were aligned by ClustalW, and the Neighbor-Joining tree was constructed using MEGA 7 with 1000 bootstrap replicates. The hollow circles represent the Arabidopsis MYB proteins; the solid black circles represent the potato R2R3-MYB subfamily proteins. The R2R3-MYB of potato and Arabidopsis were classified into 31 clades (C1-C31), PG represents PGSC0003DMG40. 


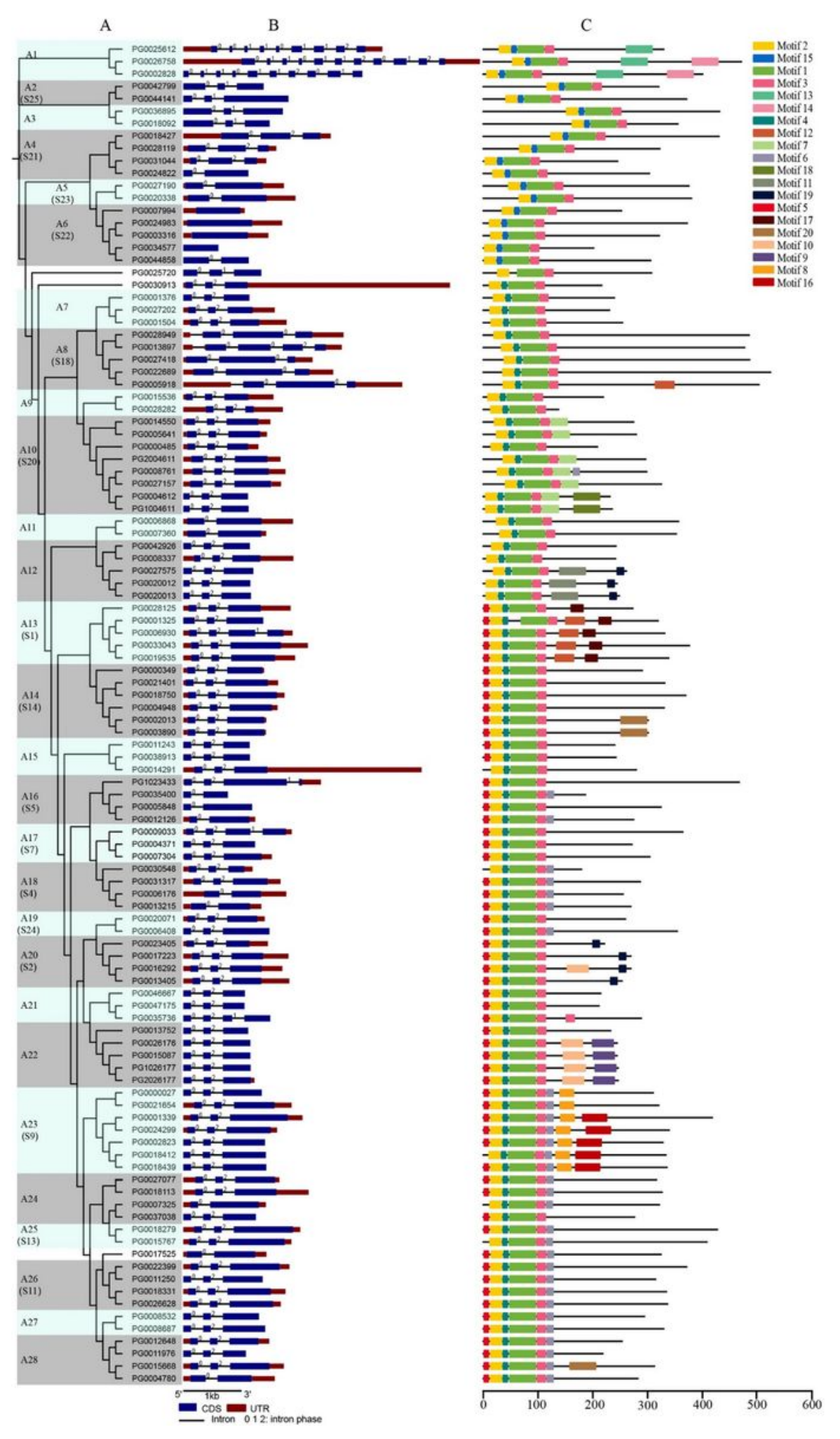

Figure 2

Phylogenetic relationships (A), gene structure (B) and motif composition (C) of StR2R3-MYB genes. A: The neighbor-joining $(\mathrm{NJ})$ tree on the left includes 108 StR2R3-MYB proteins (using ClustalW for alignment, MEGA7 for constructing the neighbor-joining tree). The MYB proteins were clustered into 28 subfamilies, sequentially designated as A1 to A28. The subfamily name which was designated as previously reports of AtMYB proteins in Arabidopsis are also marked (Dubos et al., 2010). Three proteins 
did not fit well into clusters. B: Exon/intron structures of R2R3-MYB genes from potato. Exon(s) and intron(s) are represented by blue boxes and black lines, respectively, red boxes represent 3' UTR and 5' UTR. The numbers 0,1 and 2 represent the phases of the introns. The exon-intron structures of these genes were graphically displayed by the Gene Structure Display Server using the CDS and genome sequence of StR2R3-MYB genes. C: motif compositions of StR2R3-MYB TFs. The protein sequences of StR2R3-MYB TFs were used to predict the conserved motifs by using the MEME Suite web server.
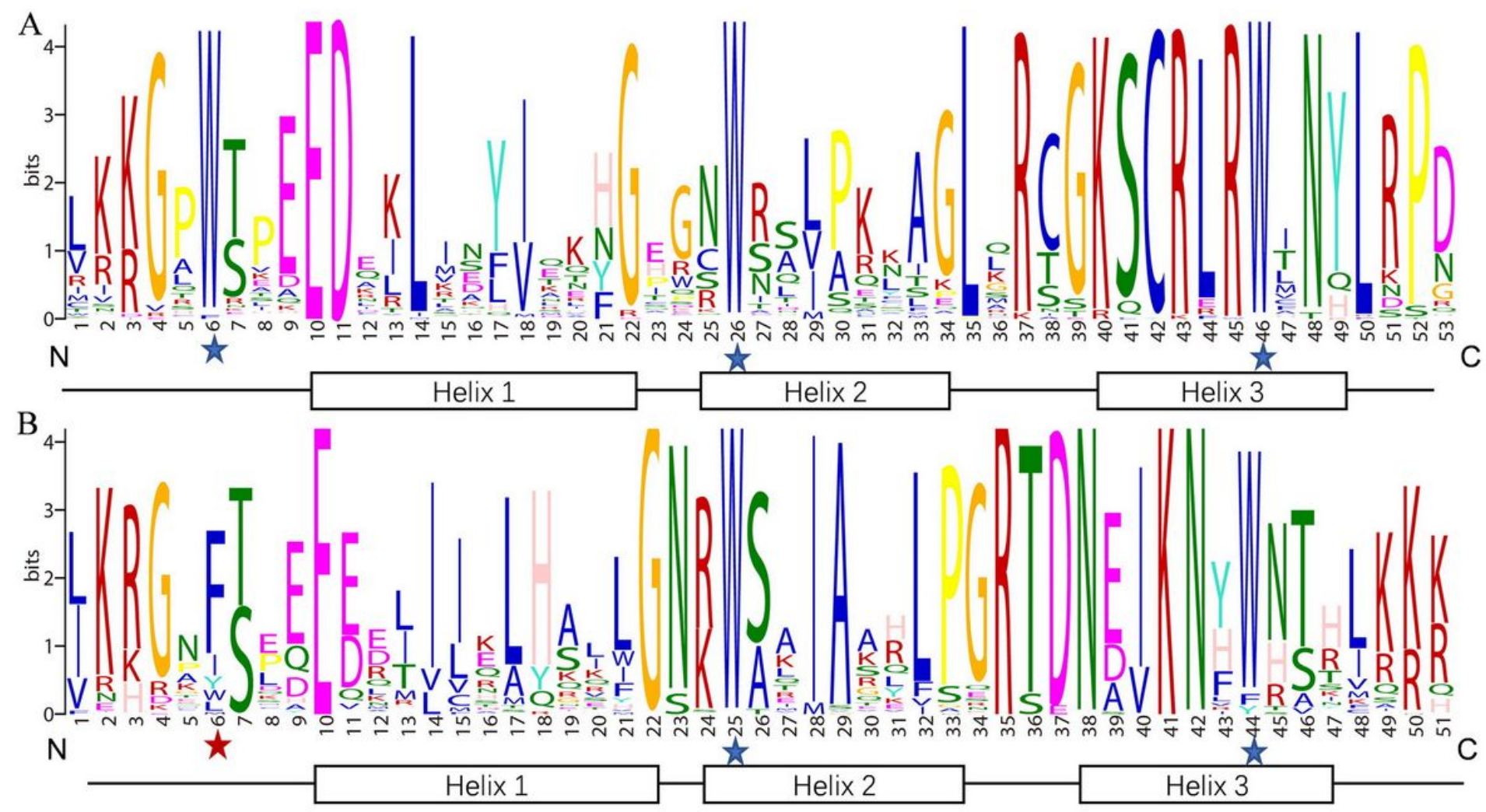

Helix 1
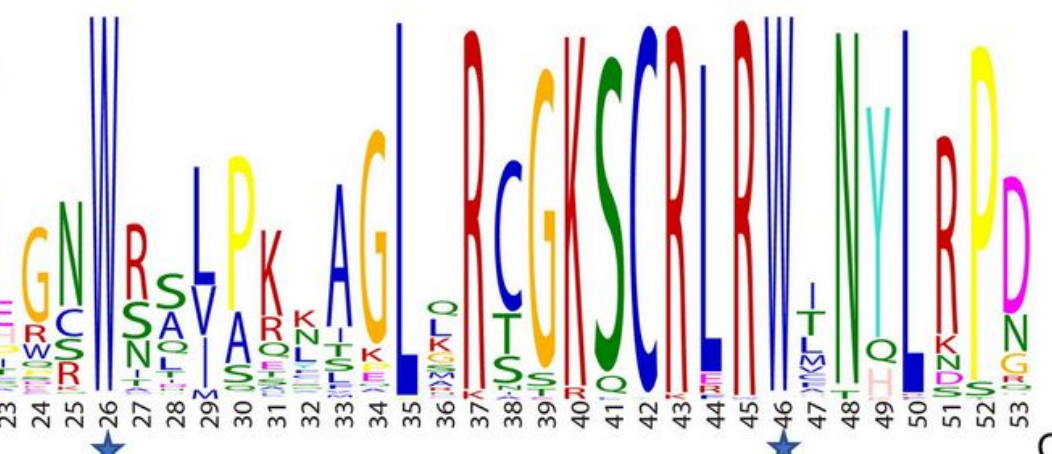

西

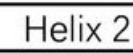

Helix 3 C

Figure 3

Sequence logos of the R2 (A) and R3 (B) MYB repeats are based on the full-length alignments of all 108 StR2R3-MYB domains. Multiple alignment analysis of the R2R3-MYB domains was performed with Clustal X. The overall height of each stack indicates the conservation of the sequence at that position and the bit score exhibits the relative frequency of the corresponding acid. The position of the three ahelices are marked (Helix 1 to 3). The conserved tryptophan residues (Trp, W) in the MYB domain are marked with blue asterisks. The replaced residues in the R3 repeat are shown by red asterisks. 
ch01

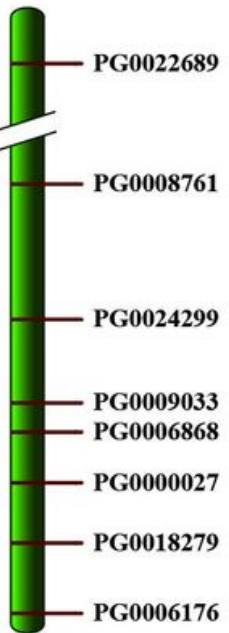

ch07

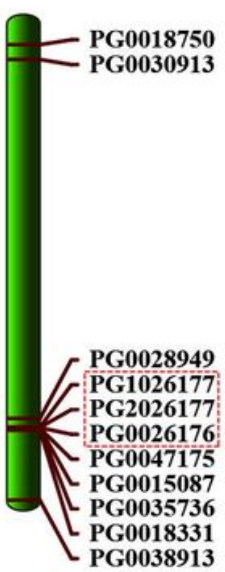

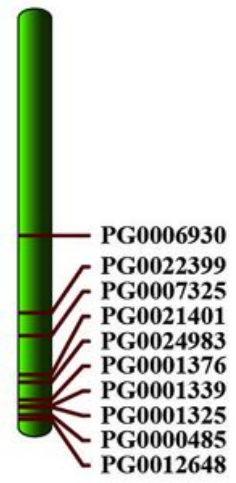

ch08

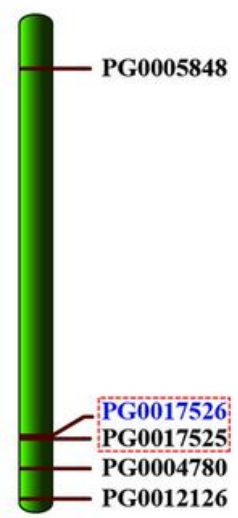

ch03

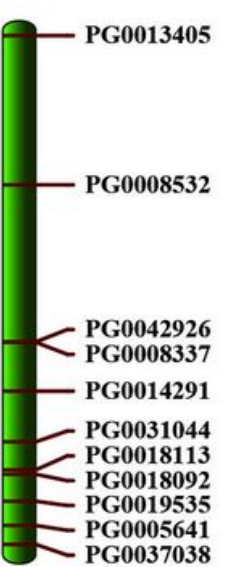

ch04

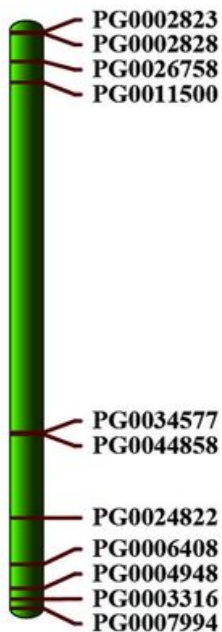

ch09

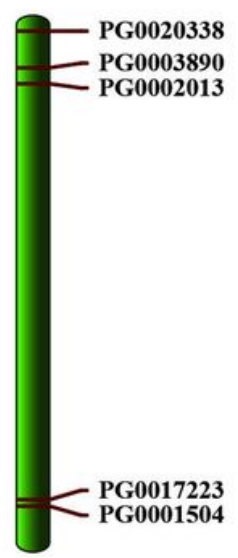

ch10

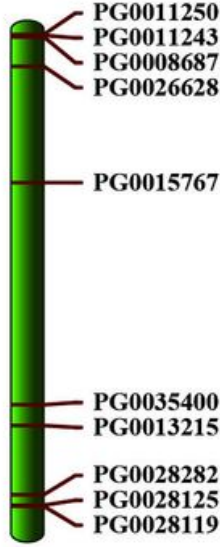

ch05

ch06

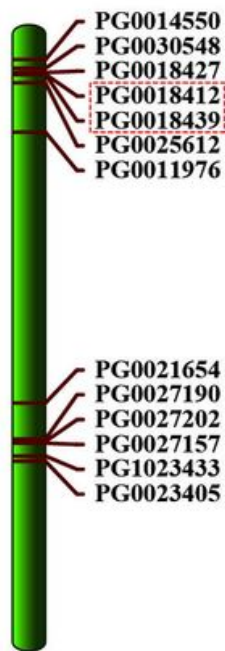

ch11

ch12

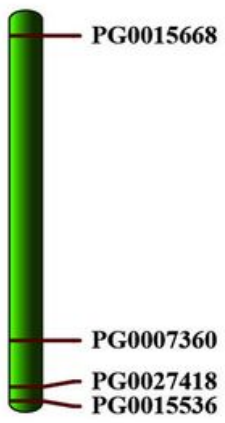

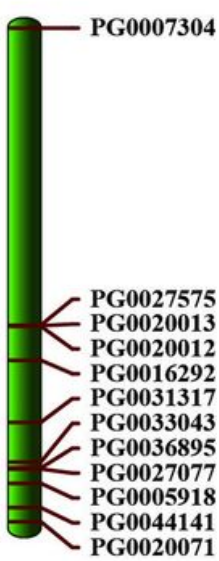

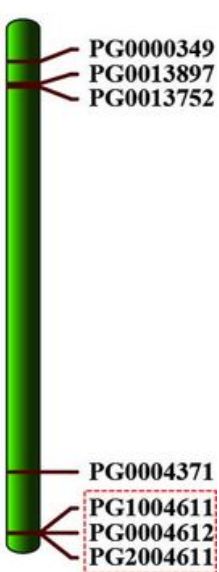

Figure 4

Chromosomal locations of potato R2R3-MYB genes. 105 out of 108 StR2R3-MYB genes are mapped to the 12 chromosomes. The chromosomal position of each StR2R3-MYB gene was mapped according to the potato genome database (PGSC). The chromosome number is indicated at the top of each chromosome. Red boxes indicate tandemly duplicated gene clusters on the chromosomes. The gene (PG0017526) marked in blue indicates a MYB-related gene. 


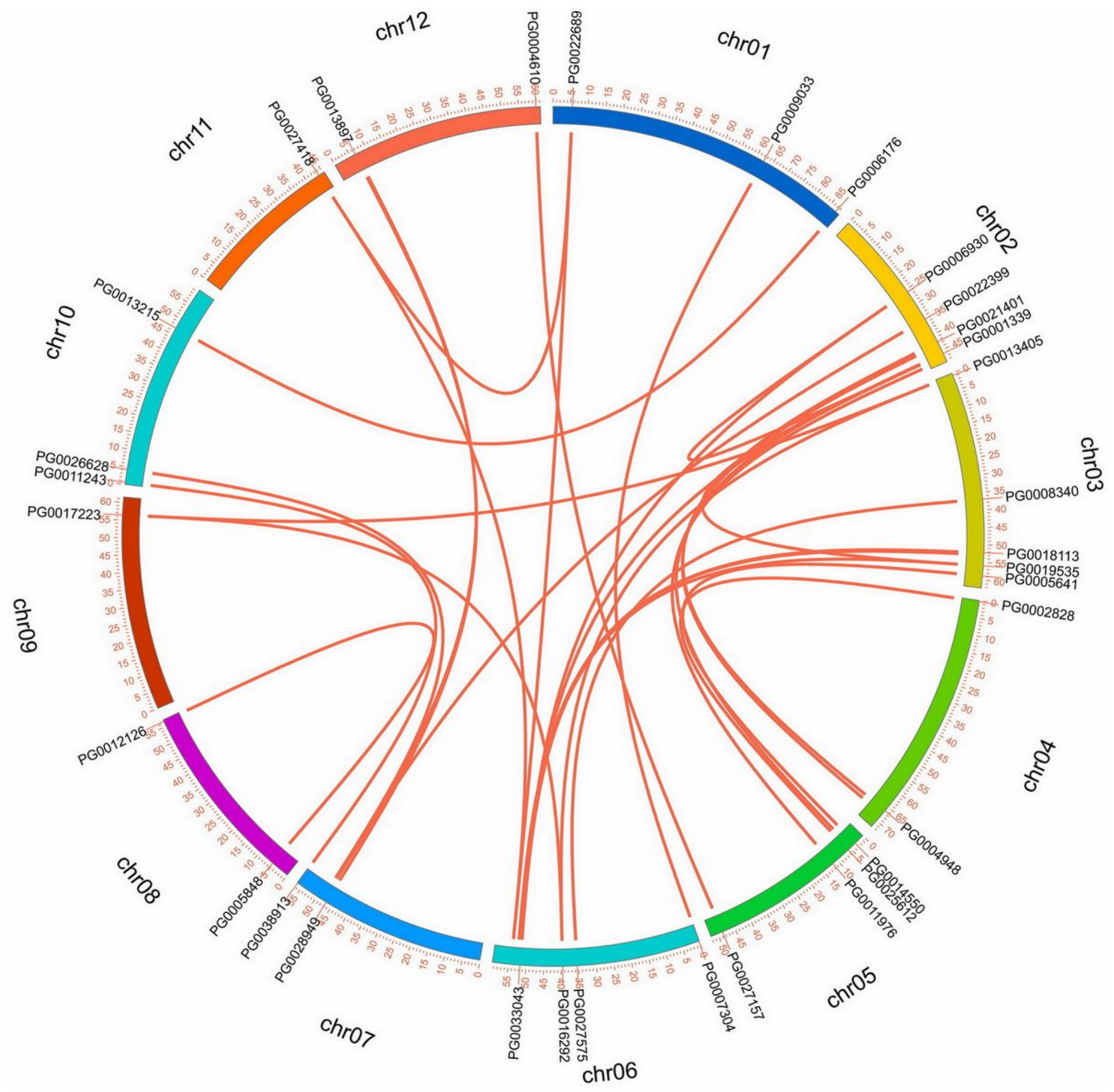

Figure 5

Genomic locations of StR2R3-MYB genes and segmentally duplicated gene pairs in the potato genome. The red lines indicate 29 segmentally duplicated R2R3-MYB gene pairs. The chromosome number is indicated at the top of each chromosome. 

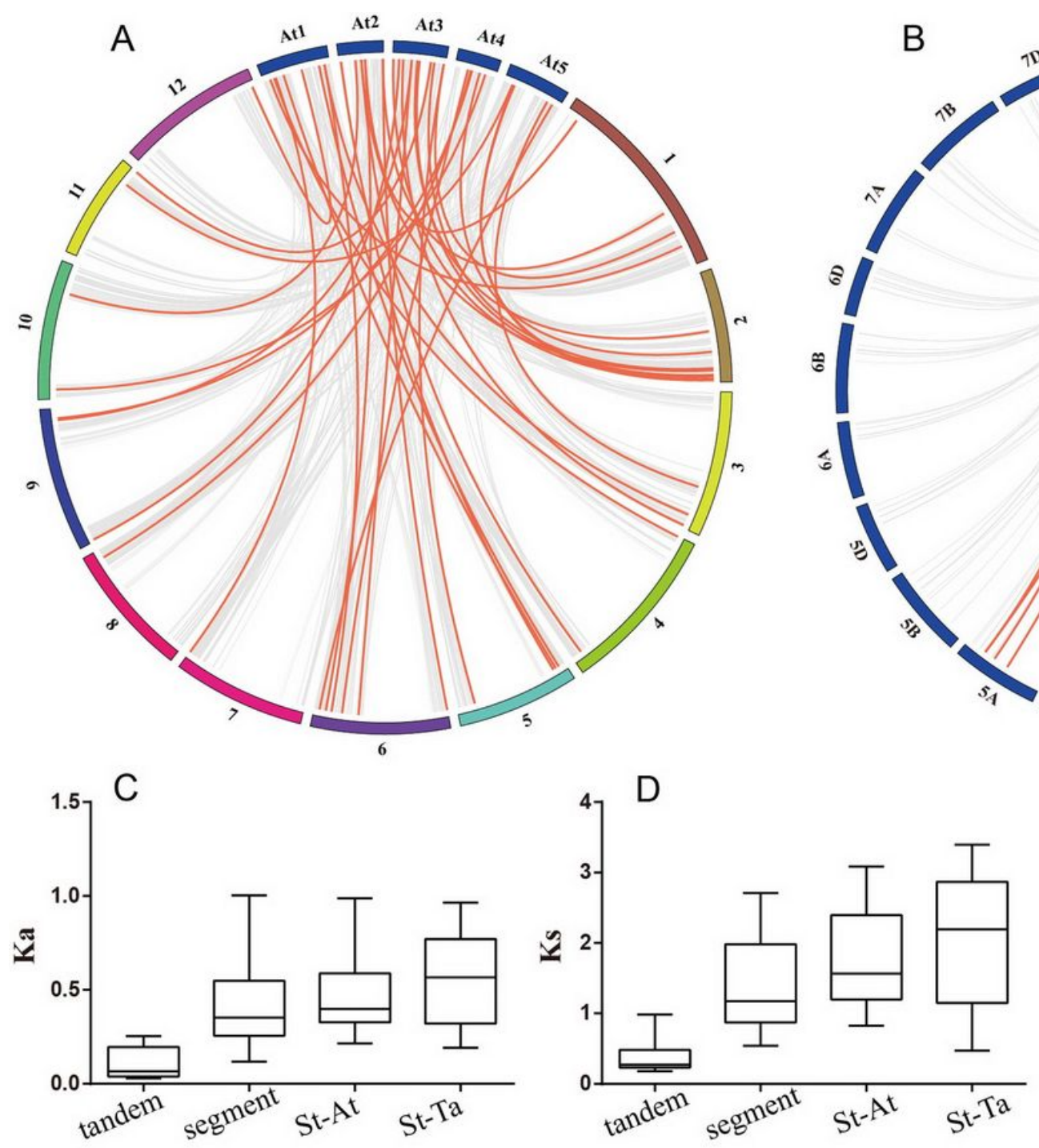
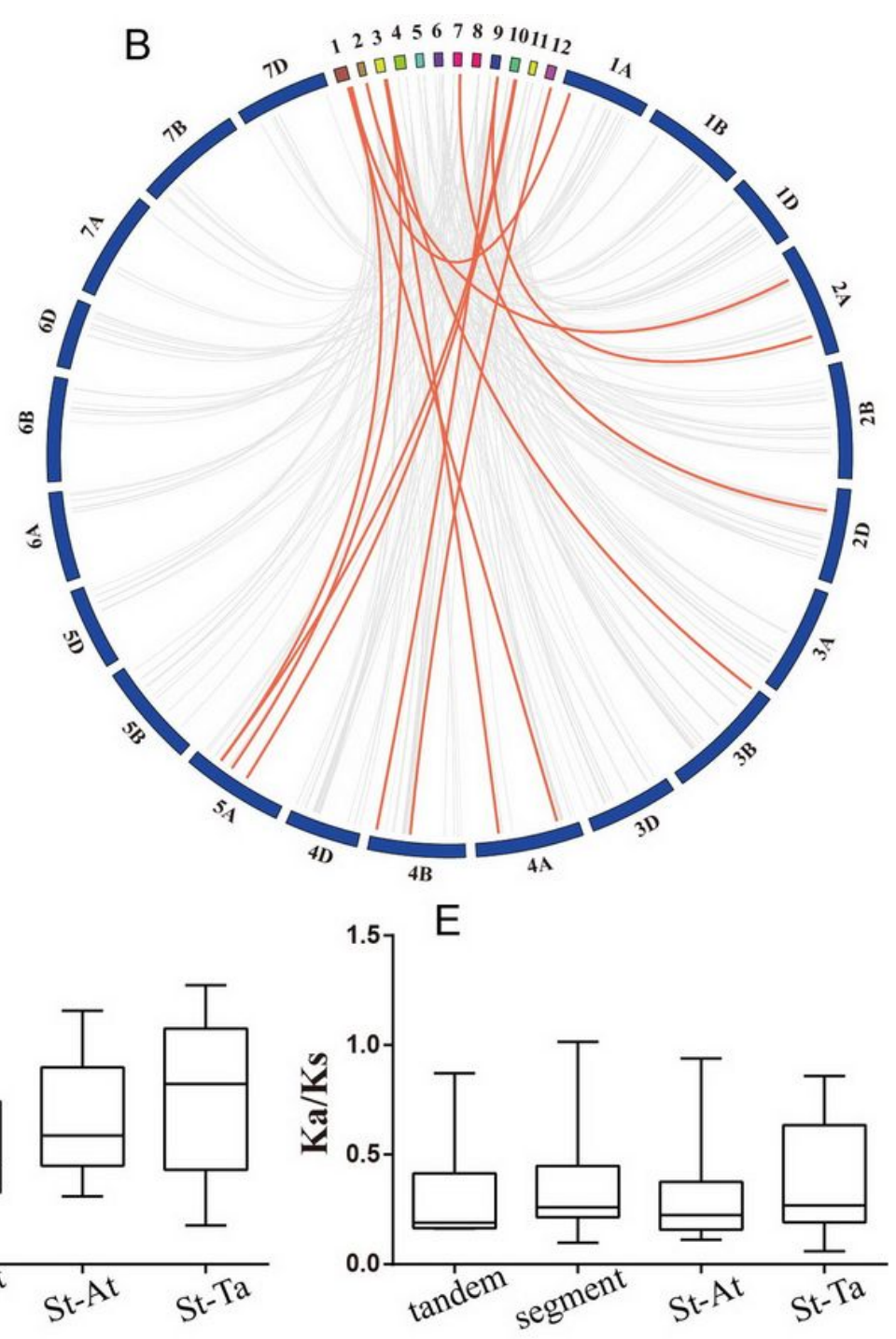

Figure 6

Comparative mapping shows the orthologous relationships of StMYB TFs with Arabidopsis (A) wheat (B). Average values of $\mathrm{Ka}, \mathrm{Ks}$ and $\mathrm{Ka} / \mathrm{Ks}$, respectively of duplicated genes were shown in $\mathrm{C}-\mathrm{E}$. The horizontal axes in (C-E) represent tandem duplication (tandem), segmental duplication (segment) and the duplication between potato and Arabidopsis (St-At) and wheat (St-Ta), respectively. 

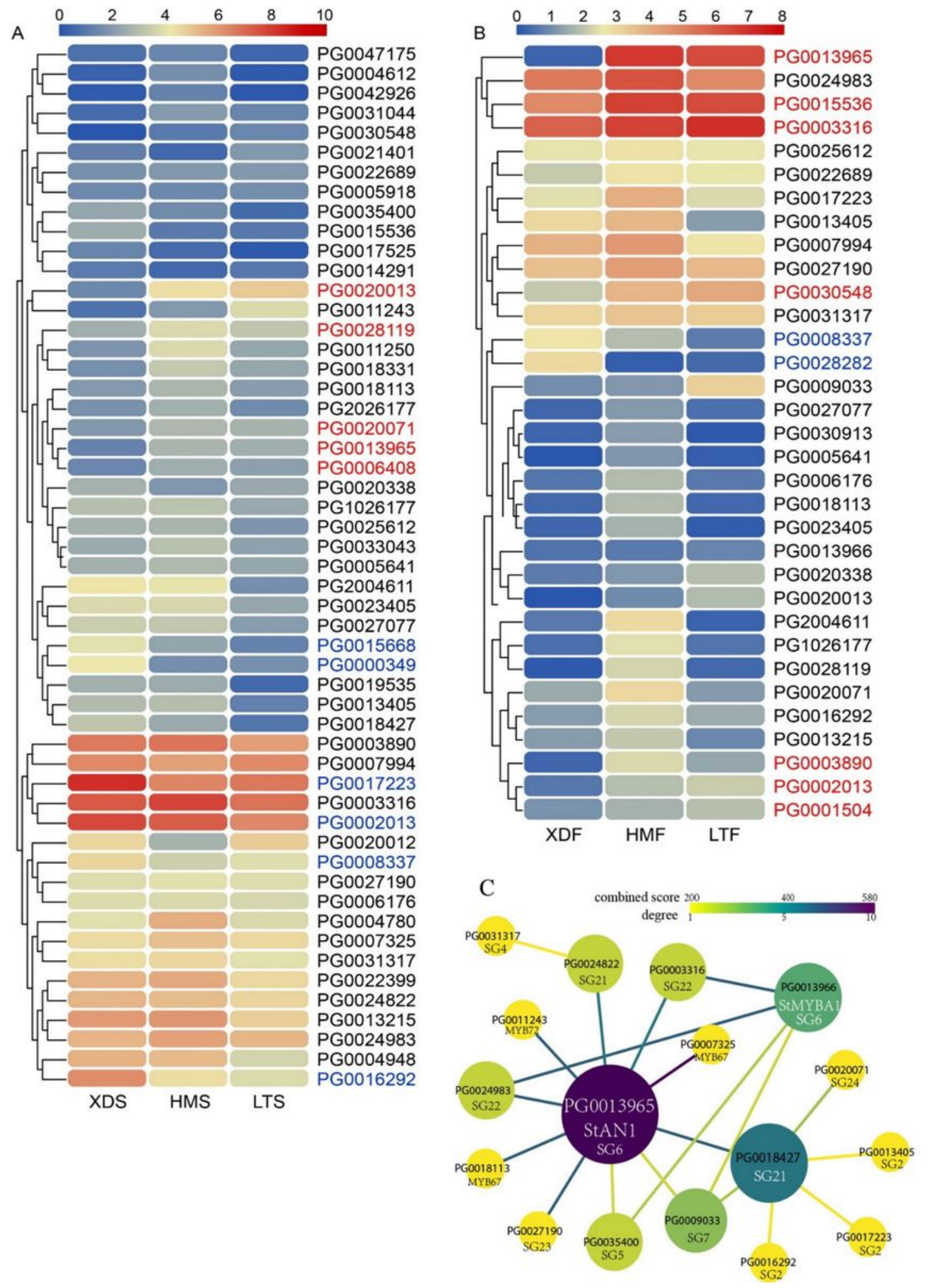

Figure 7

The expression profiles of StR2R3-MYB genes with an FPKM > 1 in white and pigmented potato tuber skin (A) and white and pigmented potato tuber flesh (B) and the interaction network of StR2R3-MYB proteins in skin and flesh (C). XDS, HMS and LTS represent white skin of white potato cultivar (XD), purple skin of purple potato cultivar (HM) and red skin of red potato cultivar (LT); XDF, HMF and LTF represent white flesh of white potato cultivar (XD), purple flesh of purple potato cultivar (HM) and red flesh of red 
potato cultivar (LT). The gene IDs marked in red represent up-regulated R2R3-MYB genes in pigmented tissues, and the gene IDs marked in blue represent up-regulated R2R3-MYB genes in white tissue. The edge color was shown from yellow to purple in accordance with the combined score (confidence) $>200$, the node size was in accordance with the node degree from yellow to purple to highlight the mostconnected genes.

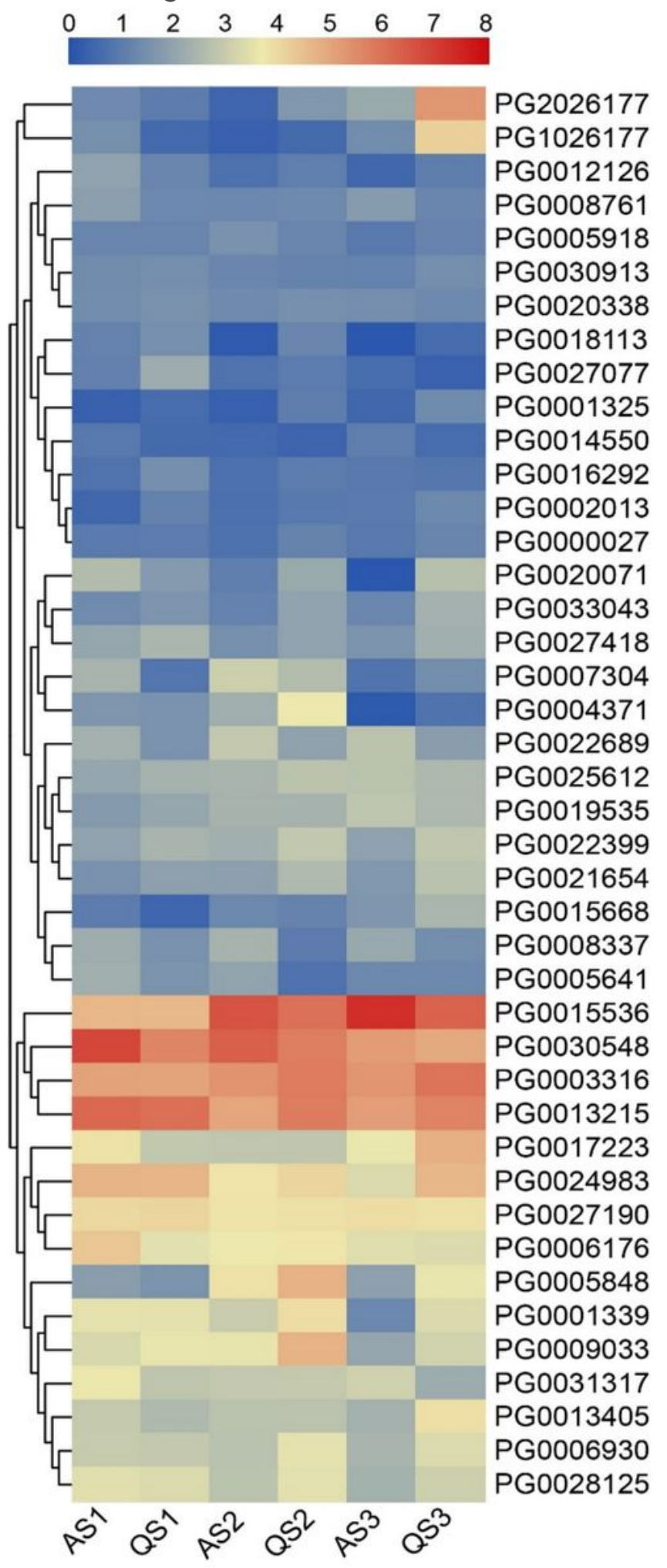

Figure 8 
The expression profiles of StR2R3-MYB genes in drought-sensitive cultivar (Atlantic, A) and droughttolerant cultivar (Qingshu No.9, Q) with an FPKM > 1 under drought stress at early flowering stage (S1), full-blooming stage (S2) and flower-falling stage (S3).

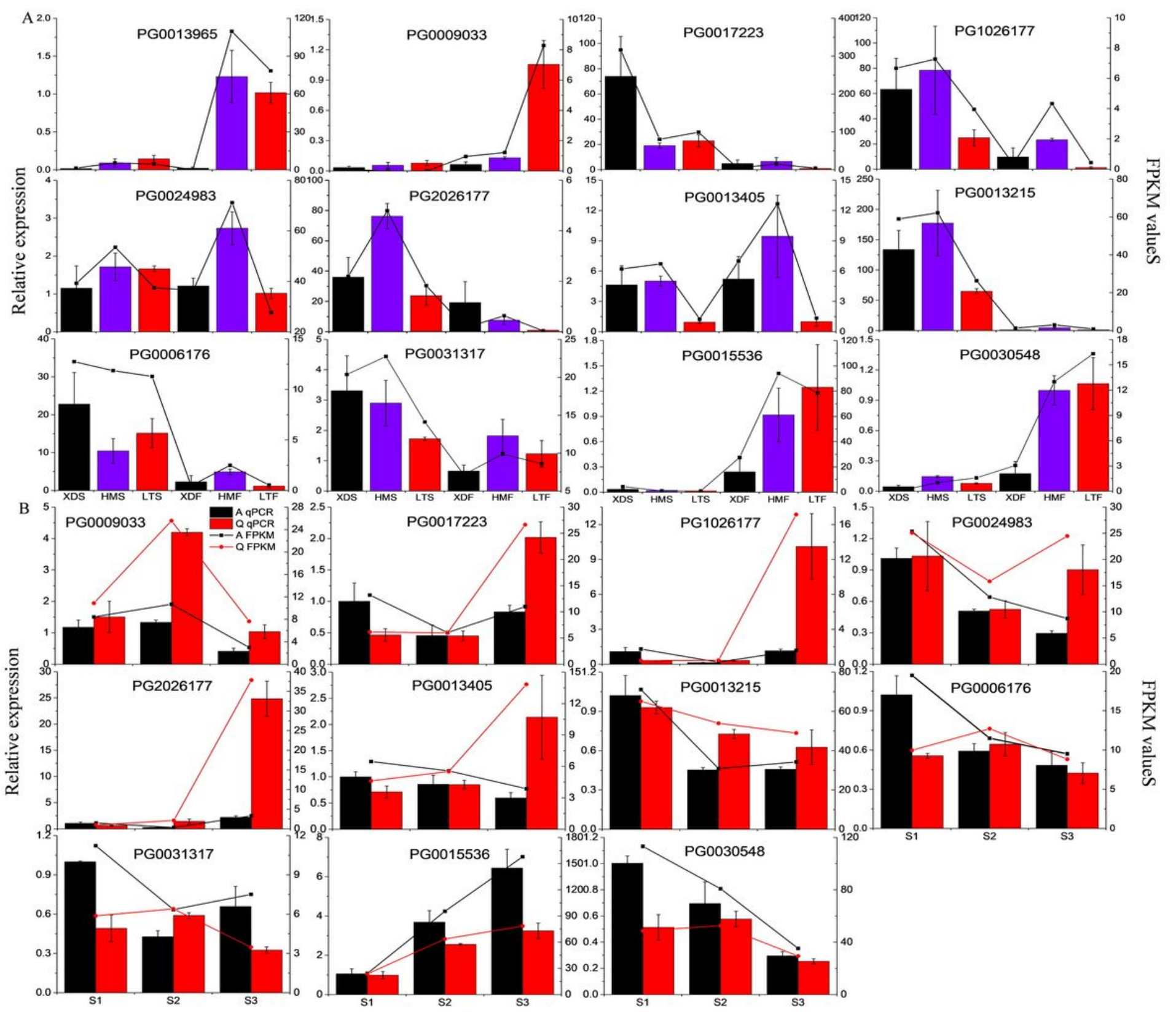

Figure 9

Quantitative RT-PCR expression analyses of 12 StR2R3-MYB genes in white and pigmented skin and flesh (A) and in drought sensitive cultivar (Atlantic, A) and drought-tolerant cultivar (Qingshu NO.9, Q) cultivar under drought stress at early flowering stage (S1), full-blooming stage (S2) and flower-falling stage (S3) (B). 


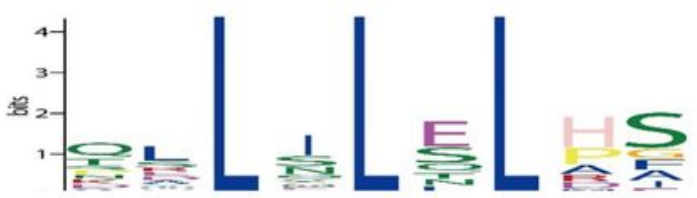

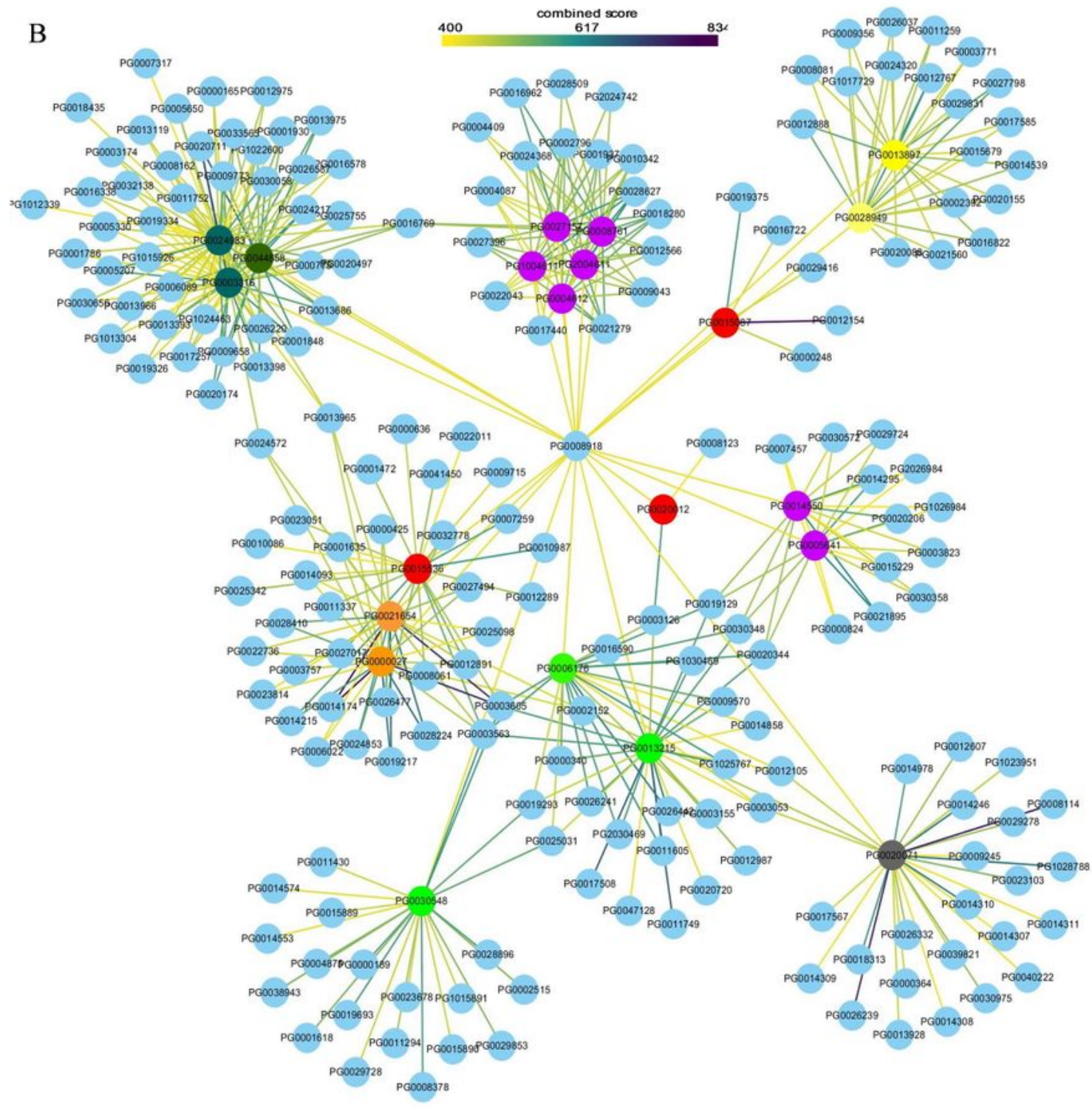

\section{Figure 10}

Protein Sequence logo of 21 EAR ( $L x L x L)$ Motifs and protein interaction network predicted for EAR motifcontaining StR2R3-MYB TFs with interacting potato proteins (combined score $>400$, medium confidence). The edge color was shown from yellow to purple in accordance with the combined score. PG0020071 in S2 was highlighted in dark grey; PG0006176, PG0013215 and PG0030548 in S4 were highlighted in green, PG0000027 and PG0021654 in S9 were highlighted in orange; PG0028949 and 
PG0013897 in S18 were highlighted in yellow; PG0008761, PG0014550, PG0027157, PG2004611, PG1004611, PG0004612 and PG0005641 in S20 were highlighted in violet; PG0044858, PG0003316 and PG0024983 in S22 were highlighted in dark green; PG0020012, PG0015087 and PG0015536 were highlighted in red.

\section{Supplementary Files}

This is a list of supplementary files associated with this preprint. Click to download.

- Tables9.xIsx

- TableS2.xlsx

- TableS1.xlsx

- FigureS1.jpg

- TableS4.xlsx

- TableS5.xIsx

- Tables8.xlsx

- FigureS2.jpg

- TableS6.xIsx

- TableS12.xlsx

- TableS11.xIsx

- Tables10.xlsx

- TableS7.xIsx

- TableS3.xlsx 